\title{
On a Nonlinear Degenerate Evolution Equation with Nonlinear Boundary Damping
}

\author{
A. T. Lourêdo, ${ }^{1}$ G. Siracusa, ${ }^{2}$ and C. A. Silva Filho ${ }^{3}$ \\ ${ }^{1}$ DM-UEPB, Campina Grande, PB, Brazil \\ ${ }^{2}$ DMA-São Cristóvão, SE, Brazil \\ ${ }^{3}$ DCET-UESC, Ilhéus, BA, Brazil \\ Correspondence should be addressed to A. T. Lourêdo; aldolouredo@gmail.com
}

Received 9 October 2014; Accepted 30 December 2014

Academic Editor: Jesus Martin-Vaquero

Copyright (C) 2015 A. T. Lourêdo et al. This is an open access article distributed under the Creative Commons Attribution License, which permits unrestricted use, distribution, and reproduction in any medium, provided the original work is properly cited.

This paper deals essentially with a nonlinear degenerate evolution equation of the form $K u^{\prime \prime}-\Delta u+\sum_{j=1}^{n} b_{j}\left(\partial u^{\prime} / \partial x_{j}\right)+|u|^{\sigma} u=0$ supplemented with nonlinear boundary conditions of Neumann type given by $\partial u / \partial v+h\left(\cdot, u^{\prime}\right)=0$. Under suitable conditions the existence and uniqueness of solutions are shown and that the boundary damping produces a uniform global stability of the corresponding solutions.

\section{Introduction}

Let $\Omega$ be a smooth bounded open set of $\mathbb{R}^{n}$, with $n \geq 1$, and its boundary $\partial \Omega=\Gamma$ of class $C^{2}$. Assume that $\Gamma$ is constituted by two disjoint closed parts $\Gamma_{0}$ and $\Gamma_{1}$ both with positive Lebesgue measure.

The main goal of this paper is to prove the existence and uniqueness as well as the uniform decay rates for the energy of the following nonlinear initial boundary value problem:

$$
\begin{gathered}
K u^{\prime \prime}-\Delta u+\sum_{j=1}^{n} b_{j} \frac{\partial u^{\prime}}{\partial x_{j}}+|u|^{\sigma} u=0 \quad \text { in } \Omega \times(0, \infty), \\
u=0 \quad \text { on } \Gamma_{0} \times(0, \infty), \\
\frac{\partial u}{\partial v}+h\left(\cdot, u^{\prime}\right)=0 \quad \text { on } \Gamma_{1} \times(0, \infty), \\
u(0, x)=u^{0}(x), \quad u^{\prime}(0, x)=u^{1}(x) \quad \text { in } \Omega,
\end{gathered}
$$

where $K=K(x, t), b_{j}=b_{j}(x, t)$, and $h=h(x, s)$ are real functions, $v=\nu(x)$ denotes the unit outward normal at $x \in$ $\Gamma_{1}$, and $\sigma>1$ is a constant.
The parabolic-hyperbolic equation $K u^{\prime \prime}-\Delta\left(u-u^{\prime}\right)+$ $f(u)=0$ when $n=1$ or $n=2$; this equation governs the motion of a nonlinear Kelvin solid. That is, a bar for $n=1$ and a plate for $n=2$, subject to no nonlinear elastic constraints. The function $K$ represents the mass density of the solid.

The existence of solutions of the linear problem associated with $(P)\left(K=1, b_{j}=0\right.$, and without the function $f(s)=|s|^{\sigma} s$ and with $h(x, s)=\delta(x) s)$ was established by Komornik and Zuazua in [1], via semigroup theory and by Milla Miranda and Medeiros in [2], applying the Galerkin's method, with a special basis. The advantage of this second method is to define the Sobolev space where $\partial u / \partial v$ is lying. In the same context, applying this second method for a wave equation with a nonlinear term, Araruna and Maciel [3], derive similar results. In Cavalcanti et al. [4] the existence of solution and an exponential decay rate is established supposing $f=0$ and $h$ being a particular function considered in our work; see also Cavalcanti et al. [5].

For the wave equation with $K=1$ and $b=0$ there is a vast literature on this problem. We cite the papers Cavalcanti et al. [6], Lasiecka and Tataru [7], and references contained therein for the reader. 
Following the ideas delivered in Milla Miranda and Medeiros [2], but bringing more technical difficulties, Milla Miranda and San Gil Jutuca [8] applied the Galerkin's method with a special basis to show the existence of solutions for Kirchhoff's equation with a linear dissipation on the boundary. Applying a similar approach, Lourêdo and Miranda [9] obtained the existence of solutions for a coupled system of Kirchhoff's equations with nonlinear boundary dissipation. For other models, but in the same context, we cite to the reader Lourêdo and Miranda [10], and Lourêdo et al. [11].

Park and Kang studied the existence, uniqueness, and uniform decay for the nonlinear degenerate equation with memory condition on the boundary in [12]. For the asymptotic behavior they also used the Nakao's method. de Lima Santos and Junior [13] studied the equation with a boundary condition with memory for Kirchhoff plates equations. An abstract formulation with the coefficient $K$ satisfying the same conditions as in our paper was studied by Pereira in [14] and was established the existence, uniqueness, and asymptotic behavior for the solutions associated with a nonlinear beam equation.

In this paper we are interested in showing the global existence of solutions for Problem $(P)$ under very general conditions to be fixed in the next section.

In our approach, we apply the Galerkin's method for a perturbed problem and a special basis; an appropriate Strauss' Lipschitz-continuous approximation $h_{l}$ of $h$; the compactness method; and results on trace mapping of nonsmooth functions. Finally, the uniform stabilization of solutions is accomplished by using the Nakao's method.

\section{Notations and Main Results}

In order to establish the main results of this paper we assume the following assumptions on the objects of problem $(P)$ :

(A1) $b_{j} \in W^{1, \infty}\left(0, \infty ; C^{1}(\bar{\Omega})\right)$ and there exists a positive constant $b_{0}>0$ such that

(A2) $\operatorname{div} b(x, t) \leq-b_{0} \forall x \in \Gamma_{1}, t \in[0, \infty)$, where $b(x, t)=\left(b_{1}(x, t), \ldots, b_{n}(x, t)\right)$;

$(H 2)$

$$
\begin{aligned}
& \frac{1}{n}<\sigma \leq \frac{2}{n-2} \quad \text { if } n \geq 3, \\
& \sigma>\frac{1}{n} \text { if } n=1,2 .
\end{aligned}
$$

(H3) $h \in C^{0}\left(\mathbb{R} ; L^{\infty}\left(\Gamma_{1}\right)\right)$ with $h: \Gamma_{1} \times \mathbb{R} \rightarrow \mathbb{R}$ strongly monotone; that is,

$$
\begin{aligned}
& {[h(x, s)-h(x, r)][s-r]} \\
& \quad \geq h_{0}(s-r)^{2} \quad \text { a.e. } x \in \Gamma_{1}, \forall s, r \in \mathbb{R},
\end{aligned}
$$

where $2 h_{0}>\|b\|_{\bar{\Omega} \times(0, \infty)}$. We use the notation $d_{0}=$ $2 h_{0}-\|b\|_{\infty}$;

(H4) $K \in C^{1}\left([0, T] ; L^{\infty}(\Omega)\right)$ with $K(x, t) \geq 0, \forall t \geq 0$, a.e. $x \in \Omega$ and there exists $\gamma>0$ such that $K(x, 0) \geq \gamma>$ 0 , a.e. $x \in \Omega$;

$(H 5)|\partial K(x, t) / \partial t|_{\mathbb{R}} \leq \delta+C(\delta) K(x, t)$, for all $\delta>0$;

(H6) $\left(u^{0}, u^{1}\right) \in D(-\Delta) \times H_{0}^{1}(\Omega)$.

The scalar product and norm of $L^{2}(\Omega)$ are denoted, respectively, by $(\cdot, \cdot)$ and $|\cdot|$. By $V$ we represent the Hilbert space $V=\left\{v \in H^{1}(\Omega) ; v=0\right.$ on $\left.\Gamma_{0}\right\}$ which is equipped with the scalar product and norm

$$
\begin{aligned}
((u, v)) & =\sum_{i=1}^{n}\left(\frac{\partial u}{\partial x_{i}}, \frac{\partial v}{\partial x_{i}}\right) \\
\|u\|^{2} & =\sum_{i=1}^{n}\left|\frac{\partial u}{\partial x_{i}}\right|^{2}
\end{aligned}
$$

The operator $-\Delta$ is defined by the triplet $\left\{V, L^{2}(\Omega),((\cdot, \cdot))\right\}$. Then its domain is given by

$$
D(-\Delta)=\left\{u \in V \cap H^{2}(\Omega) ; \frac{\partial u}{\partial \nu}=0 \text { on } \Gamma_{1}\right\} .
$$

From spectral theory it follows that $D(-\Delta)$ is dense in $V$; see [15]. Moreover, it will be denoted

$$
\begin{aligned}
(u, v)_{L^{2}\left(\Gamma_{1}\right)} & =\int_{\Gamma_{1}} u(x) v(x) d \Gamma, \\
|u|_{L^{2}\left(\Gamma_{1}\right)}^{2} & =\int_{\Gamma_{1}} u^{2}(x) d \Gamma, \\
\|u\|_{\infty} & =\text { ess } \sup _{t \geq 0}\|u(t)\|_{L^{\infty}(\Omega)} .
\end{aligned}
$$

Theorem 1. Assume hypotheses (H1)-(H6); there exists at least a function $u: \Omega \times(0, \infty) \rightarrow \mathbb{R}$ in the class

$$
\begin{aligned}
& u \in L_{\mathrm{loc}}^{\infty}(0, \infty ; V), \\
& u^{\prime} \in L_{\mathrm{loc}}^{\infty}(0, \infty ; V), \\
& u^{\prime \prime} \in L_{\mathrm{loc}}^{\infty}\left(0, \infty ; L^{2}(\Omega)\right), \\
& \Delta u \in L_{\mathrm{loc}}^{\infty}\left(0, \infty ; L^{2}(\Omega)\right), \\
& \frac{\partial u}{\partial v} \in L_{\mathrm{loc}}^{1}\left(0, \infty ; L^{1}\left(\Gamma_{1}\right)\right),
\end{aligned}
$$


satisfying

$$
\begin{gathered}
K u^{\prime \prime}-\Delta u+\sum_{j=1}^{n} b_{j} \frac{\partial u^{\prime}}{\partial x_{j}}+|u|^{\sigma} u=0 \\
\text { in } L_{\text {loc }}^{\infty}\left(0, \infty ; L^{2}(\Omega)\right), \\
\frac{\partial u}{\partial v}+h\left(\cdot, u^{\prime}\right)=0 \quad \text { in } L_{\text {loc }}^{1}\left(0, \infty ; L^{1}\left(\Gamma_{1}\right)\right), \\
u(0)=u^{0}, \quad u^{\prime}(0)=u^{1} \quad \text { in } \Omega .
\end{gathered}
$$

In addition, if

(H7) $|h(x, s)| \leq h_{1}|s| \forall s \in \mathbb{R}$ and a.e. $x$ in $\Gamma_{1}\left(h_{1}\right.$ a positive constant), holds true, we have the following result.

Corollary 2. Under the hypothesis of Theorem 1 and (H7), the solution $u$ of Problem $(P)$ (obtained in Theorem 1) is unique and has the following regularity:

$$
\begin{gathered}
u \in L_{\mathrm{loc}}^{\infty}(0, \infty ; V) \cap L_{\mathrm{loc}}^{2}\left(0, \infty ; H^{3 / 2}(\Omega)\right), \\
u^{\prime} \in L_{\mathrm{loc}}^{\infty}(0, \infty ; V), \\
u^{\prime \prime} \in L_{\mathrm{loc}}^{\infty}\left(0, \infty ; L^{2}(\Omega)\right), \\
K u^{\prime \prime}-\Delta u+\sum_{j=1}^{n} b_{j} \frac{\partial u^{\prime}}{\partial x_{j}}+|u|^{\sigma} u=0 \\
\text { in } L_{\mathrm{loc}}^{\infty}\left(0, \infty ; L^{2}(\Omega)\right), \\
\frac{\partial u}{\partial v}+h\left(\cdot, u^{\prime}\right)=0 \quad \text { in } L_{\mathrm{loc}}^{2}\left(0, \infty ; L^{2}\left(\Gamma_{1}\right)\right), \\
u(0)=u^{0}, \quad u^{\prime}(0)=u^{1} \quad \text { in } \Omega .
\end{gathered}
$$

Remark 3. If we replace the function $f(s)=|s|^{\sigma} s$ in Theorem 1 by a continuous function $g$ such that

$$
\begin{aligned}
& {[g(s)-g(r)](s-r)} \\
& \quad \geq g_{0}(s-r)^{2}, \quad \forall s, r \in \mathbb{R},\left(g_{0} \text { a positive constant }\right),
\end{aligned}
$$

and further

$$
|g(s)| \leq g_{1}|s|, \quad \forall s \in \mathbb{R}
$$

Theorem 1 remains valid. Indeed, from (10), we obtain

$$
\left|g_{l}(s)\right| \leq g_{1}|s|, \quad \forall s \in \mathbb{R}
$$

where $\left(g_{l}\right)$ is the Strauss' approximations (see [16]) of the function $g$.

Remark 4. Analogously if $|h(x, s)| \leq h_{1}|s| \forall s \in \mathbb{R}$ and a.e. $x$ in $\Gamma_{1}\left(h_{1}\right.$ a positive constant), we obtain $\left|h_{l}(x, s)\right| \leq(3 / 2) h_{1}|s|$ for all $s \in \mathbb{R}$ (see $[10])$.
For use later, note that

$$
\begin{aligned}
& |w|^{2} \leq \frac{1}{\lambda_{1}}\|w\|^{2}, \\
& \|w\|_{L^{2}\left(\Gamma_{1}\right)} \leq k_{1}\|w\|,
\end{aligned}
$$

$\forall w \in V, \quad\left(k_{1}\right.$ positive constants $)$;

where $\lambda_{1}$ is the first eigenvalue of the spectral problem $((u$, $v))=\lambda(u, v)$ for all $v \in V$ (see [15]).

In order to establish the uniform decay rate for energy, we assume

(H8) $\left\|K^{\prime}\right\|_{\infty}<b_{0} / 2$;

(H9) $\|K\|_{\infty}=$ ess $\sup _{t \geq 0}\|K(t)\|_{L^{\infty}(\Omega)}$;

$(H 10)\|\operatorname{div} b\|_{\infty} \leq \delta_{0}$;

$(H 11)\|b\|_{\infty}<(21 / 32) \lambda_{1}, b_{0}>\left(32\left(1 / \lambda_{1}\right) / M\right)\|K\|_{\infty},\|K\|_{\infty}>$ $\|b\|_{\infty} / 2+8\left(1 / \lambda_{1}\right) / M$, where $M=\min \{1,1-(9 / 32+$ $\left.\left.\left(1 / \lambda_{1}\right)\|b\|_{\infty}\right)\right\}$.

Remark 5. There are functions that satisfy hypothesis $(H 5)$ and (H8). In fact, the function $K(x, t)=\left(\alpha(x) /\|\alpha\|_{\infty}\right) \beta(t)$ with $\alpha \in C^{1}(\bar{\Omega}), \alpha(x) \geq 0$, and $\beta(t)=\left(b_{0} / 4\right) e^{-t}$ satisfies such hypothesis, since

$$
\left|K^{\prime}(x, t)\right| \leq \frac{\alpha(x)}{\|\alpha\|_{\infty}} \frac{b_{0}}{4} e^{-t} \leq \frac{b_{0}}{4}<\frac{b_{0}}{2},
$$

and so

$$
\left|K^{\prime}(x, t)\right| \leq \delta+K(x, t), \quad \forall \delta \geq 0 .
$$

Theorem 6. Under the hypothesis of Theorem 1 and (H7)(H11), with $h_{1}(s)$ satisfying (9) and (10), the energy

$$
\begin{aligned}
E(t)=\frac{1}{2}\left\{K^{1 / 2}(t)\left|u^{\prime}(t)\right|^{2}+\|u(t)\|^{2}\right. & \\
& \left.+\frac{2}{\sigma+2} \int_{\Omega}|u(t)|^{\sigma+2} d x\right\} \quad \text { for } t \geq 1,
\end{aligned}
$$

associated with the solution, $u$, obtained in Corollary 2 is uniformly stable; that is, there exists a positive constant such that

$$
E(t) \leq C \exp (-\omega t), \quad \forall t \geq 1,
$$

where $C$ and $\omega$ are positive constants.

For use later, we observe that hypothesis in $(H 3)_{1}$ on $\sigma$ implies $q^{*}=2 n /(n-2) \geq 2 \sigma+2$ and $q^{*} \geq \sigma n$. Thus, the Sobolev's embedding gives

$$
\begin{aligned}
& V \hookrightarrow L^{q^{*}}(\Omega) \hookrightarrow L^{2 \sigma+2}(\Omega) \quad \text { for } n \geq 3, \\
& V \hookrightarrow L^{2 \sigma+2}(\Omega) \quad \text { for } n=1,2, \\
& V \hookrightarrow L^{q^{*}}(\Omega) \hookrightarrow L^{\sigma n}(\Omega) \quad \text { for } n \geq 3, \\
& V \hookrightarrow L^{\sigma n}(\Omega) \quad \text { for } n=1,2 .
\end{aligned}
$$


Here, $X \hookrightarrow Y$ indicates that the subspace $X$ is continuously embedded in the space $Y$.

Next, following the ideas contained in Strauss [16], we approximate the function $h$ by Lipschitz-continuous ones $h_{l}$.

\section{Proof of Theorem 1}

For our purposes we need the following previous results, whose proof can be seen in [10].

Lemma 7. Let $h$ be a function satisfying hypothesis (H3). Then there exists a sequence $\left(h_{l}\right)$ of functions in $C^{0}\left(\mathbb{R} ; L^{\infty}\left(\Gamma_{1}\right)\right)$ such that

(i) $h_{l}(x, 0)=0$ a.e. $x$ in $\Gamma_{1}$;

(ii) $\left[h_{l}(x, s)-h_{l}(x, r)\right](s-r) \geq h_{0}(s-r)^{2}, \forall s, r \in \mathbb{R}$, and a.e. $x$ in $\Gamma_{1}$; ( $h_{0}$ positive constant);

(iii) for any $l$ there exists a function $c_{l}$ in $L^{\infty}\left(\Gamma_{1}\right)$ satisfying

$$
\begin{aligned}
& \left|h_{l}(x, s)-h_{l}(x, r)\right| \\
& \quad \leq c_{l}|s-r|, \quad \forall s, r \in \mathbb{R} \text {, a.e. } x \text { in } \Gamma_{1} ;
\end{aligned}
$$

(iv) $\left(h_{l}\right)$ converges to g uniformly on bounded sets on $\mathbb{R}$ and a.e. $x$ in $\Gamma_{1}$.

Lemma 8. Let $T>0$ be a real number. Consider the sequence $\left(w_{l}\right)$ of vectors in $L^{2}\left(0, T ; H^{-1 / 2}\left(\Gamma_{1}\right)\right) \cap L^{1}\left(0, T ; L^{1}\left(\Gamma_{1}\right)\right)$ and vectors $w \in L^{2}\left(0, T ; H^{-1 / 2}\left(\Gamma_{1}\right)\right)$ and $\chi \in L^{1}\left(0, T ; L^{1}\left(\Gamma_{1}\right)\right)$ such that

(i) $w_{l} \rightarrow w$ weak in $L^{2}\left(0, T ; H^{-1 / 2}\left(\Gamma_{1}\right)\right)$;

(ii) $w_{l} \rightarrow \chi$ in $L^{1}\left(0, T ; L^{1}\left(\Gamma_{1}\right)\right)$.

Then, $w=\chi$.

Lemma 9. Let $p: \mathbb{R} \rightarrow \mathbb{R}$ be a globally Lipschitz-continuous function with $p(0)=0$ and let

$$
\gamma_{0}: V \longrightarrow H^{1 / 2}\left(\Gamma_{1}\right)
$$

be the continuous trace of order zero. Consider $u \in L^{2}(0, T ; V)$ then $p(u) \in L^{2}(0, T ; V), p\left(\gamma_{0} u\right) \in L^{2}\left(0, T ; H^{1 / 2}\left(\Gamma_{1}\right)\right)$, and $\gamma_{0} p(u(t))=p\left(\gamma_{0} u(t)\right)$ a.e. $t \in(0, T)$.

Proof. We see that

$$
\begin{aligned}
p: V & \longrightarrow V, \\
p: H^{1 / 2}\left(\Gamma_{1}\right) & \longrightarrow H^{1 / 2}\left(\Gamma_{1}\right)
\end{aligned}
$$

are continuous maps (see Brezis and Cazenave [17] and Marcus and Mizel [18]). Let $v \in V$. Consider a sequence $\left(v_{k}\right)$ of functions of $C^{1}(\bar{\Omega})$ such that

$$
v_{k} \longrightarrow v \text { in } V .
$$

Then by (21) and (22), we have $p\left(v_{k}\right) \rightarrow p(v)$ in $V$, and by (20)

$$
\gamma_{0} p\left(v_{k}\right) \longrightarrow \gamma_{0} p(v) \quad \text { in } H^{1 / 2}\left(\Gamma_{1}\right)
$$

Also by (20) and (22), we deduce

$$
p\left(\gamma_{0} v_{k}\right) \longrightarrow p\left(\gamma_{0} v\right) \quad \text { in } H^{1 / 2}\left(\Gamma_{1}\right)
$$

As $\gamma_{0} p\left(v_{k}\right)=p\left(\gamma_{0} v_{k}\right)$, it follows from (23) and (24) that $\gamma_{0} p(v)=p\left(\gamma_{0} v\right)$. This implies

$$
\gamma_{0} p(u(t))=p\left(\gamma_{0} u(t)\right) \quad \text { a.e. in }(0, T)
$$

Now, we consider the set $\mathcal{O}=\{s \in \mathbb{R}: p$ is not differentiable in $s\}$. Then

$$
\begin{aligned}
& \frac{\partial}{\partial x_{i}} p(u(x, t)) \\
& \quad= \begin{cases}p^{\prime}(u(x, t)) \frac{\partial u}{\partial x_{i}}(x, t), & u(x, t) \notin \mathcal{O}, \\
0, & u(x, t) \in \mathcal{O},\end{cases}
\end{aligned}
$$

with $i=1,2, \ldots, n$ (see Brezis and Cazenave, loc. cit). As $p^{\prime} \in$ $L^{\infty}(\mathbb{R})$ then

$$
p(u) \in L^{2}(0, T ; V) \text {. }
$$

From this and since $\gamma_{0}: L^{2}(0, T ; V) \rightarrow L^{2}\left(0, T ; H^{1 / 2}\left(\Gamma_{1}\right)\right)$ then $\gamma_{0} p(u) \in L^{2}\left(0, T ; H^{1 / 2}\left(\Gamma_{1}\right)\right)$. This and (25) furnish

$$
p\left(\gamma_{0} u\right) \in L^{2}\left(0, T ; H^{1 / 2}\left(\Gamma_{1}\right)\right) \text {. }
$$

From (25) to (28) we have the results of this Lemma.

Proof of Theorem 1. We will use the Faedo-Galerkin's method with a special basis of $V \cap H^{2}(\Omega)$. Thus, let us consider the Strauss' approximation $\left(h_{l}\right)$ of $h$ given by Lemma 7 . Let us consider $\left(u_{l}^{1}\right)$ a sequence of vectors in $\mathscr{D}(\Omega)$ such that

$$
u_{l}^{1} \longrightarrow u^{1} \quad \text { in } H_{0}^{1}(\Omega)
$$

Note that $h_{l}\left(x, u_{l}^{1}\right)=0$ and $\partial u^{0} / \partial \nu=0$ on $\Gamma_{1}$ since $u^{0} \epsilon$ $D(-\Delta)$. Thus,

$$
\frac{\partial u^{0}}{\partial \nu}+h_{l}\left(\cdot, u_{l}^{1}\right)=0 \quad \text { on } \Gamma_{1}, \forall l
$$

Now, we fix $l$ and construct the basis $\left\{w_{1}^{l}, w_{2}^{l}, \ldots,\right\}$ of $V \cap$ $H^{2}(\Omega)$ such that $u^{0}, u_{l}^{1}$ belong to the subspace $\left[w_{1}^{l}, w_{2}^{l}\right]$ spanned by $w_{1}^{l}$ and $w_{2}^{l}$. Let $V_{m}^{l}=\left[w_{1}^{l}, w_{2}^{l}, \ldots, w_{m}^{l}\right]$ be the subspace of $V \cap H^{2}(\Omega)$ spanned by $w_{1}^{l}, \ldots, w_{m}^{l}$. With this basis 
we determine the approximate solutions $u_{\varepsilon l m}(t)$ of Problem $(A P)$, where $0<\varepsilon<1$ fixed.

Approximated Perturbed Problem. This consists to find the functions $u_{\varepsilon l m}(t)=\sum_{j=1}^{m} g_{\varepsilon j l m}(t) w_{j}^{l}$, solutions of the problem

$$
\begin{gathered}
\left((K(t)+\varepsilon) u_{\varepsilon l m}^{\prime \prime}(t), w_{j}^{l}\right)+\left(\left(u_{\varepsilon l m}(t), w_{j}^{l}\right)\right) \\
+\left(\sum_{j=1}^{n} b_{j}(t) \frac{\partial u_{\varepsilon l m}^{\prime}(t)}{\partial x_{j}}, w_{j}^{l}\right) \\
+\left(\left|u_{\varepsilon l m}(t)\right|^{\sigma} u_{\varepsilon l m}(t), w_{j}^{l}\right) \\
+\left(h_{l}\left(\cdot, u_{\varepsilon l m}^{\prime}(t)\right), w_{j}^{l}\right)_{L^{2}\left(\Gamma_{1}\right)}=0, \quad j=1,2, \ldots, m, \\
u_{\varepsilon l m}(0)=u^{0}, \\
u_{\varepsilon l m}^{\prime}(0)=u_{l}^{1} .
\end{gathered}
$$

The above finite-dimensional system has a solution, $u_{\varepsilon l m}(t)$, defined on $\left[0, t_{s l m}[\right.$. The following estimate allows us to extend this solution to the whole interval $[0, \infty)$.

\subsection{Estimates}

3.1.1. First Estimate. Considering $w=2 u_{\varepsilon l m}^{\prime}(t)$ in $(A P)_{1}$, integrating from 0 to $t\left(0 \leq t<t_{\varepsilon l m}\right)$, using the fact that $h_{l}(x, s) s \geq h_{0} s^{2}$ (see Part (ii) of Lemma 7), assumptions (H4) and (H5), and since $0<\varepsilon<1$, we obtain

$$
\begin{aligned}
&(K(t)\left., u_{\varepsilon l m}^{\prime 2}(t)\right)+\varepsilon\left|u_{\varepsilon l m}^{\prime}(t)\right|^{2}+\left\|u_{\varepsilon l m}(t)\right\|^{2} \\
&+\frac{2}{\sigma+2} \int_{\Omega}\left|u_{\varepsilon l m}(t)\right|^{\sigma+2} d x \\
&+2 h_{0} \int_{0}^{t} \int_{\Gamma_{1}}\left(u_{\varepsilon l m}^{\prime}(x, s)\right)^{2} d \Gamma d s \\
&+2 \int_{0}^{t} \sum_{j=1}^{n} \int_{\Omega} b_{j}(s) \frac{\partial u_{\varepsilon l m}^{\prime}(s)}{\partial x_{j}} u_{\varepsilon l m}^{\prime}(s) d x d s \\
& \leq\left|u_{l}^{1}\right|^{2}+|| u^{0} \|^{2}+|K(0)|\left|u_{l}^{1}\right|^{2}+\frac{2}{\sigma+2} \int_{\Omega}\left|u^{0}\right|^{\sigma+2} d x \\
& \quad+\int_{0}^{t}\left[\delta\left|u_{\varepsilon l m}^{\prime}(s)\right|^{2}+C(\delta)\left(K(s), u_{\varepsilon l m}^{\prime 2}(s)\right)\right] d s .
\end{aligned}
$$

Note that

$$
\begin{aligned}
\sum_{j=1}^{n} \int_{\Omega} b_{j}(t) \frac{\partial u_{\varepsilon l m}^{\prime}}{\partial x_{j}}(t) u_{\varepsilon l m}^{\prime}(t) d x= & -\frac{1}{2} \int_{\Omega} \operatorname{div}(b)\left|u_{\varepsilon l m}^{\prime}\right|^{2} d x \\
& +\frac{1}{2} \int_{\Gamma_{1}} b \cdot \nu\left|u_{\varepsilon l m}^{\prime}\right|^{2} d \Gamma .
\end{aligned}
$$

In fact, by the Gauss's formula we have

$$
\begin{aligned}
\int_{\Omega} b_{j} u_{\varepsilon l m}^{\prime} \frac{\partial u_{\varepsilon l m}^{\prime}}{\partial x_{j}} d x= & -\frac{1}{2} \int_{\Omega} \frac{\partial b_{j}}{\partial x_{j}}\left|u_{\varepsilon l m}^{\prime}\right|^{2} d x \\
& +\frac{1}{2} \int_{\Gamma_{1}} b_{j} v_{j}\left|u_{\varepsilon l m}^{\prime}\right|^{2} d \Gamma,
\end{aligned}
$$

where $v_{j}$ is the $j$ th entry of the normal vector $v$. Hence, by $(A 2)$ we obtain

$$
\begin{aligned}
& \int_{0}^{t} \sum_{j=1}^{n} \int_{\Omega} b_{j} u_{\varepsilon l m}^{\prime} \frac{\partial u_{\varepsilon l m}^{\prime}(s)}{\partial x_{j}} d x d s \\
& \geq \frac{b_{0}}{2} \int_{0}^{t} \int_{\Omega}\left|u_{\varepsilon l m}^{\prime}(s)\right|^{2} d x d s \\
& \quad+\frac{1}{2} \int_{0}^{t} \int_{\Gamma_{1}} b(s) \cdot v\left|u_{\varepsilon l m}^{\prime}(t)\right|^{2} d \Gamma d s .
\end{aligned}
$$

Using the hypothesis $\operatorname{div} b(t) \leq-b_{0}$, choosing $\delta=b_{0} / 4>0$, and plugging (34) in (31), we find

$$
\begin{aligned}
(K(t) & \left., u_{\varepsilon l m}^{\prime 2}(t)\right)+\varepsilon\left|u_{\varepsilon l m}^{\prime}(t)\right|^{2}+\left\|u_{\varepsilon l m}(t)\right\|^{2} \\
& +\frac{b_{0}}{2} \int_{0}^{t}\left|u_{\varepsilon l m}^{\prime}(s)\right|^{2} d s+\frac{2}{\sigma+2} \int_{\Omega}\left|u_{\varepsilon l m}(t)\right|^{\sigma+2} d x \\
& +d_{0} \int_{0}^{t} \int_{\Gamma_{1}}\left(u_{\varepsilon l m}^{\prime}(x, s)\right)^{2} d \Gamma d s \\
\leq & c_{0}\left|u^{1}\right|^{2}+\left\|u^{0}\right\|^{2}+c_{1}\left\|u^{0}\right\|^{\sigma+2} \\
& +C(\delta) \int_{0}^{t}\left(K(s), u_{\varepsilon l m}^{\prime 2}(s)\right) d s,
\end{aligned}
$$

where $d_{0}=2 h_{0}-\|b\|_{\infty} / 2>0$ for all $l \geq l_{0}, \forall m$ and $0<\varepsilon<1$. Moreover, $\|v\|_{L^{\sigma+2}(\Omega)} \leq c_{1}\|v\|$ for all $v \in V$. Therefore by the Gronwall's inequality and (35)

$$
\begin{gathered}
\left(K^{1 / 2} u_{\varepsilon l m}^{\prime}\right) \text { is bounded in } L_{\mathrm{loc}}^{\infty}\left(0, \infty ; L^{2}(\Omega)\right), \\
\left(u_{\varepsilon l m}\right) \text { is bounded in } L_{\mathrm{loc}}^{\infty}(0, \infty ; V), \\
\left(u_{\varepsilon l m}^{\prime}\right) \text { is bounded in } L_{\mathrm{loc}}^{2}\left(0, \infty ; L^{2}\left(\Gamma_{1}\right)\right) .
\end{gathered}
$$

3.1.2. Second Estimate. Differentiating with respect to $t$ the approximate equation $(A P)_{1}$ and taking $w=2 u_{\varepsilon l m}^{\prime \prime}(t)$, we obtain

$$
\begin{aligned}
& \frac{d}{d t}\left(K(t) u_{\varepsilon l m}^{\prime \prime}(t), u_{\varepsilon l m}^{\prime \prime}(t)\right)+\frac{d}{d t} \varepsilon\left|u_{\varepsilon l m}^{\prime \prime}(t)\right|^{2} \\
& \quad+\frac{d}{d t}\left\|u_{\varepsilon l m}^{\prime}(t)\right\|^{2}+\left(K^{\prime}(t) u_{\varepsilon l m}^{\prime \prime}(t), u_{\varepsilon l m}^{\prime \prime}(t)\right) \\
& \quad+2 \int_{\Gamma_{1}} h_{l}^{\prime}\left(\cdot, u_{\varepsilon l m}^{\prime}(t)\right)\left|u_{\varepsilon l m}^{\prime \prime}(t)\right|^{2} d \Gamma
\end{aligned}
$$


6

Journal of Applied Mathematics

$$
\begin{aligned}
& +2 \sum_{i=1}^{n}\left(b_{i} \frac{\partial u_{\varepsilon l m}^{\prime \prime}(t)}{\partial x_{i}}, u_{\varepsilon l m}^{\prime \prime}(t)\right) \\
& +2(\sigma+1)\left(\left|u_{\varepsilon l m}(t)\right|^{\sigma} u_{\varepsilon l m}^{\prime}(t), u_{\varepsilon l m}^{\prime \prime}(t)\right) \\
& +2 \sum_{i=1}^{n}\left(b_{i}^{\prime}(t) \frac{\partial u_{\varepsilon l m}^{\prime}(t)}{\partial x_{i}}(t), u_{\varepsilon l m}^{\prime \prime}(t)(t)\right)=0 .
\end{aligned}
$$

Note that

$$
\begin{aligned}
& \sum_{j=1}^{n}\left(b_{j}(t) \frac{\partial u_{\varepsilon l m}^{\prime \prime}(t)}{\partial x_{j}}(t), u_{\varepsilon l m}^{\prime \prime}(t)\right) \\
& \quad=-\frac{1}{2} \int_{\Omega} \operatorname{div}(b)\left|u_{\varepsilon l m}^{\prime \prime}(t)\right|^{2} d x+\frac{1}{2} \int_{\Gamma_{1}} b(t) \cdot v\left|u_{\varepsilon l m}^{\prime \prime}(t)\right|^{2} d \Gamma
\end{aligned}
$$

and that

$$
\begin{aligned}
& \left|\sum_{j=1}^{n}\left(b_{j}^{\prime}(t) \frac{\partial u_{\varepsilon l m}^{\prime}(t)}{\partial x_{j}}, u_{\varepsilon l m}^{\prime \prime}(t)\right)\right| \\
& \quad \leq n \max _{1 \leq j \leq n}\left\|b_{j}^{\prime}\right\|_{\infty}\left|\frac{\partial u_{\varepsilon l m}^{\prime}}{\partial x_{j}}(t)\right|\left|u_{\varepsilon l m}^{\prime \prime}(t)\right| \\
& \quad \leq n \max _{1 \leq j \leq n}\left\|b_{j}^{\prime}\right\|_{\infty}\left|\nabla u_{\varepsilon l m}^{\prime}(t)\right|\left|u_{\varepsilon l m}^{\prime \prime}(t)\right| \\
& \quad \leq \frac{n^{1 / 2} \max _{1 \leq j \leq n}\left\|b_{j}^{\prime}\right\|_{\infty}}{2} C_{\rho}\left|\nabla u_{\varepsilon l m}^{\prime}(t)\right|^{2}+\rho\left|u_{\varepsilon l m}^{\prime \prime}(t)\right|^{2}
\end{aligned}
$$

where the last inequality becomes from Young's inequality. Therefore, if

$$
K_{2}:=C_{\rho} \frac{n^{1 / 2} \max _{1 \leq j \leq n}\left\|b_{j}\right\|_{\infty}}{2},
$$

then $\left|\sum_{j=1}^{n}\left(b_{j}^{\prime}(t)\left(\partial u_{\varepsilon l m}^{\prime}(t) / \partial x_{j}\right)(t), u_{\varepsilon l m}^{\prime \prime}(t)\right)\right| \leq K_{2}\left\|u_{\varepsilon l m}^{\prime}(t)\right\|^{2}+$ $\rho\left|u_{\varepsilon l m}^{\prime \prime}(t)\right|^{2}$. Combining this inequality and (38) with (37), after that using that $h_{l}^{\prime}(\cdot, s) \geq h_{0}$, $\operatorname{div} b(t) \leq-b_{0}$ and integrating from 0 to $t$, we obtain

$$
\begin{aligned}
(K(t) & \left., u_{\varepsilon l m}^{\prime \prime 2}(t)\right)+\varepsilon\left|u_{\varepsilon l m}^{\prime \prime}(t)\right|^{2}+\left\|u_{\varepsilon l m}^{\prime}(t)\right\|^{2} \\
& +\left(\frac{b_{0}}{2}-\rho\right) \int_{0}^{t}\left|u_{\varepsilon l m}^{\prime \prime}(s)\right|^{2} d s \\
& +\left(2 h_{0}-\frac{\|b\|_{\infty}}{2}\right) \int_{0}^{t} \int_{\Gamma_{1}}\left|u_{\varepsilon l m}^{\prime \prime}(s)\right|^{2} d \Gamma d s \\
\leq \mid & \left.u_{\varepsilon l m}^{\prime \prime}(0)\right|^{2}+\left\|u_{l}^{1}\right\|^{2}+\left|K^{1 / 2}(0)\right|\left|u_{\varepsilon l m}^{\prime \prime}(0)\right| \\
& -2(\sigma+1) \int_{0}^{t}\left(\left|u_{\varepsilon l m}(s)\right|^{\sigma} u_{\varepsilon l m}^{\prime}(s), u_{\varepsilon l m}^{\prime \prime}(s)\right) d s
\end{aligned}
$$

$$
\begin{aligned}
& +\delta \int_{0}^{t}\left|u_{\varepsilon l m}^{\prime \prime}(s)\right|^{2} d s+K_{2} \int_{0}^{t}\left\|u_{\varepsilon l m}^{\prime}(s)\right\|^{2} \\
& +\int_{0}^{t} C(\delta)\left(K(s), u_{\varepsilon l m}^{\prime \prime 2}(s)\right) d s .
\end{aligned}
$$

We also have used the above hypotheses $(H 4)$ and (H5). Now, by Hölder's inequality for $1 / q_{1}^{*}+1 / n+1 / 2=1$, embedding (18) and first estimate (36), we find

$$
\begin{aligned}
& \left|\left(\left|u_{\varepsilon l m}(t)\right|^{\sigma} u_{\varepsilon l m}^{\prime}(t), u_{\varepsilon l m}^{\prime \prime}(t)\right)\right| \\
& \leq\left(\int_{\Omega}\left|u_{\varepsilon l m}(t)\right|^{\sigma n}\right)^{1 / n}\left(\int_{\Omega}\left|u_{\varepsilon l m}^{\prime}(t)\right|^{q_{1}^{*}}\right)^{1 / q_{1}^{*}} \\
& \cdot\left(\int_{\Omega}\left|u_{\varepsilon l m}^{\prime \prime}(t)\right|^{2}\right)^{1 / 2} \\
& \leq C\left\|u_{\varepsilon l m}(t)\right\|^{\sigma}\left\|u_{\varepsilon l m}^{\prime}(t)\right\|\left|u_{\varepsilon l m}^{\prime \prime}(t)\right| \\
& \leq C\left\|u_{\varepsilon l m}^{\prime}(t)\right\|\left|u_{\varepsilon l m}^{\prime \prime}(t)\right| \\
& \leq C_{\eta}\left\|u_{l m}^{\prime}(t)\right\|^{2}+\eta\left|u_{l m}^{\prime \prime}(t)\right|^{2},
\end{aligned}
$$

where the constant $C$ is independent of $l, m, \varepsilon$ and $t \geq 0$, and $C_{\eta}$ is a positive constant that depends on $\eta$. Substituting this inequality in (41) yields

$$
\begin{aligned}
(K(t) & \left.u_{\varepsilon l m}^{\prime \prime}(t), u_{\varepsilon l m}^{\prime \prime}(t)\right)+\varepsilon\left|u_{\varepsilon l m}^{\prime \prime}(t)\right|^{2} \\
& +\left\|u_{\varepsilon l m}^{\prime}(t)\right\|^{2}\left(\frac{b_{0}}{2}-(\rho+\eta)\right) \int_{0}^{t}\left|u_{\varepsilon l m}^{\prime \prime}(s)\right|^{2} d s \\
& +\left(2 h_{0}-\frac{\|b\|_{\infty}}{2}\right) \int_{0}^{t} \int_{\Gamma_{1}}\left|u_{\varepsilon l m}^{\prime \prime}(s)\right|^{2} d \Gamma d s \\
\leq \mid & \left.u_{\varepsilon l m}^{\prime \prime}(0)\right|^{2}+\left\|u_{l}^{1}\right\|^{2}+\sup _{x \in \Omega} K(x, 0)\left|u_{\varepsilon l m}^{\prime \prime}(0)\right|^{2} \\
& +K_{2} \int_{0}^{t}\left\|u_{\varepsilon l m}^{\prime}(s)\right\|^{2}+\delta \int_{0}^{t}\left|u_{\varepsilon l m}^{\prime \prime}(s)\right|^{2} d s \\
& +\int_{0}^{t} C(\delta)\left(K(s) u_{\varepsilon l m}^{\prime \prime}(s), u_{\varepsilon l m}^{\prime \prime}(s)\right) d s .
\end{aligned}
$$

Choosing $\delta=b_{0} / 4$ and $\rho, \eta$ small such that $b_{1}=\left(b_{0} / 2-(\rho+\right.$ $\eta))>0$, we find

$$
\begin{aligned}
& \left(K(t), u_{\varepsilon l m}^{\prime \prime 2}(t)\right)+\varepsilon\left|u_{\varepsilon l m}^{\prime \prime}(t)\right|^{2}+\left\|u_{\varepsilon l m}^{\prime}(t)\right\|^{2} \\
& \quad+b_{1} \int_{0}^{t}\left|u_{\varepsilon l m}^{\prime \prime}(s)\right|^{2} d s+d_{0} \int_{0}^{t} \int_{\Gamma_{1}}\left|u_{\varepsilon l m}^{\prime \prime}(s)\right|^{2} d \Gamma d s \\
& \leq\left|u_{\varepsilon l m}^{\prime \prime}(0)\right|^{2}+\left\|u_{l}^{1}\right\|^{2}+\sup _{x \in \Omega} K(x, 0)\left|u_{\varepsilon l m}^{\prime \prime}(0)\right|^{2} \\
& \quad+K_{2} \int_{0}^{t}\left\|u_{\varepsilon l m}^{\prime}(s)\right\|^{2}+C(\delta)\left(K(s) u_{\varepsilon l m}^{\prime \prime}(s), u_{\varepsilon l m}^{\prime \prime}(s)\right) d s
\end{aligned}
$$


Now we need to derive an estimate for $\left(u_{\varepsilon l m}^{\prime \prime}(0)\right)$. Thus, taking $t=0$ in approximate Problem $(A P)_{1}$ and choosing $v=$ $u_{\varepsilon l m}^{\prime \prime}(0)$, one has

$$
\begin{aligned}
& \left((K(0)+\varepsilon) u_{\varepsilon l m}^{\prime \prime}(0), u_{\varepsilon l m}^{\prime \prime}(0)\right)+\left(\left(u^{0}, u_{\varepsilon l m}^{\prime \prime}(0)\right)\right) \\
& \quad+\int_{\Gamma_{1}} h_{l}\left(\cdot, u_{l}^{1}\right) u_{\varepsilon l m}^{\prime \prime}(0) d \Gamma+\sum_{i=1}^{n}\left(b_{j}(0) \frac{\partial u_{l}^{1}}{\partial x_{i}}, u_{\varepsilon l m}^{\prime \prime}(0)\right) \\
& \quad+\left(\left|u^{0}\right|^{\sigma} u^{0}, u_{\varepsilon l m}^{\prime \prime}(0)\right)=0 .
\end{aligned}
$$

Applying Green's formula

$$
\begin{aligned}
& \left((K(0)+\varepsilon) u_{\varepsilon l m}^{\prime \prime}(0), u_{\varepsilon l m}^{\prime \prime}(0)\right)-\left(\Delta u^{0}, u_{\varepsilon l m}^{\prime \prime}(0)\right) \\
& \quad+\int_{\Gamma_{1}}\left[\frac{\partial u^{0}}{\partial \nu}+h_{l}\left(\cdot, u_{l}^{1}\right)\right] u_{\varepsilon l m}^{\prime \prime}(0) d \Gamma \\
& \quad+\sum_{i=1}^{n}\left(b_{j}(0) \frac{\partial u_{l}^{1}}{\partial x_{i}}, u_{\varepsilon l m}^{\prime \prime}(0)\right)+\left(\left|u^{0}\right|^{\sigma} u^{0}, u_{\varepsilon l m}^{\prime \prime}(0)\right)=0 .
\end{aligned}
$$

Thanks to (30), the integrals on $\Gamma_{1}$ in (46) are null. Using convergence (29), embedding (17), and the hypothesis (H4) give

$$
\left|u_{\varepsilon l m}^{\prime \prime}(0)\right| \leq C, \quad \forall l, m, \varepsilon
$$

From this boundedness and (46), we get

$$
\begin{aligned}
& \left(K(t) u_{\varepsilon l m}^{\prime \prime}(t), u_{\varepsilon l m}^{\prime \prime}(t)\right)+\varepsilon\left|u_{\varepsilon l m}^{\prime \prime}(t)\right|^{2}+\left\|u_{\varepsilon l m}^{\prime}(t)\right\|^{2} \\
& \quad+b_{1} \int_{0}^{t}\left|u_{\varepsilon l m}^{\prime \prime}(s)\right|^{2} d s+d_{0} \int_{0}^{t} \int_{\Gamma_{1}}\left|u_{\varepsilon l m}^{\prime \prime}(s)\right|^{2} d \Gamma d s \\
& \leq C_{1}+K_{2} \int_{0}^{t}\left\|u_{\varepsilon l m}^{\prime}(s)\right\|^{2} \\
& +\int_{0}^{t} C(\delta)\left(K(s) u_{\varepsilon l m}^{\prime \prime}(t), u_{\varepsilon l m}^{\prime \prime}(s)\right) d s,
\end{aligned}
$$

where $C_{1}$ is constant independent of $l, m$, and $\varepsilon$. Applying Gronwall's inequality in (48) and using estimate (47), we have

$$
\begin{gathered}
\left(K^{1 / 2} u_{\varepsilon l m}^{\prime \prime}\right) \text { is bounded in } L_{\text {loc }}^{\infty}\left(0, \infty ; L^{2}(\Omega)\right) ; \\
\left(u_{\varepsilon l m}^{\prime \prime}\right) \text { is bounded in } L_{\text {loc }}^{2}\left(0, \infty ; L^{2}(\Omega)\right) ; \\
\left(u_{\varepsilon l m}^{\prime}\right) \text { is bounded in } L_{\text {loc }}^{\infty}(0, \infty ; V) ; \\
\left(u_{\varepsilon l m}^{\prime \prime}\right) \text { is bounded in } L_{\text {loc }}^{2}\left(0, \infty ; L^{2}\left(\Gamma_{1}\right)\right) .
\end{gathered}
$$

From estimates (36), (49), induction, and diagonal process, we obtain a subsequence of $\left(u_{\varepsilon l m}\right)$, which is still denoted by $\left(u_{\varepsilon l m}\right)$, and a function $\left.u_{\varepsilon l}: \Omega \times\right] 0, \infty[\rightarrow \mathbb{R}$, such that

$$
\begin{gathered}
u_{\varepsilon l m} \longrightarrow u_{\epsilon l} \text { weak star in } L_{\mathrm{loc}}^{\infty}(0, \infty ; V) ; \\
u_{\varepsilon l m}^{\prime} \longrightarrow u_{\varepsilon l}^{\prime} \text { weak star in } L_{\mathrm{loc}}^{\infty}(0, \infty ; V) ; \\
K u_{\varepsilon l m}^{\prime \prime} \longrightarrow K u_{\epsilon l}^{\prime \prime} \text { weak star in } L_{\mathrm{loc}}^{\infty}\left(0, \infty ; L^{2}(\Omega)\right) ; \\
u_{\varepsilon l m}^{\prime} \longrightarrow u_{\varepsilon l}^{\prime} \text { weak in } L_{\mathrm{loc}}^{2}\left(0, \infty ; L^{2}\left(\Gamma_{1}\right)\right) ; \\
u_{\varepsilon l m}^{\prime \prime} \longrightarrow u_{\varepsilon l}^{\prime \prime} \text { weak in } L_{\mathrm{loc}}^{2}\left(0, \infty ; L^{2}\left(\Gamma_{1}\right)\right) .
\end{gathered}
$$

From the convergence $(49)_{2}$ we obtain

$$
\begin{aligned}
& \sum_{i=1}^{n} b_{j} \cdot \frac{\partial u_{\varepsilon l m}^{\prime}}{\partial x_{j}} \\
& \quad \longrightarrow \sum_{i=1}^{n} b_{j} \cdot \frac{\partial u_{\varepsilon l}^{\prime}}{\partial x_{j}} \text { weak star in } L_{\text {loc }}^{\infty}\left(0, \infty ; L^{2}(\Omega)\right)
\end{aligned}
$$

3.1.3. Analysis of the Nonlinear Terms. By estimates (36) $)_{1}$, $(36)_{2}$, compactness method (cf. Lions [19] or Simon [20]), embedding (17), induction, and diagonal process, we obtain a subsequence of $\left(u_{\varepsilon l m}\right)$, which also is denoted by $\left(u_{\varepsilon l m}\right)$, such that

$$
\begin{aligned}
& \left|u_{\varepsilon l m}\right|^{\sigma} u_{l m} \\
& \quad \longrightarrow\left|u_{\varepsilon l}\right|^{\sigma} u_{\varepsilon l} \text { weak star in } L_{\text {loc }}^{\infty}\left(0, \infty ; L^{2}(\Omega)\right) .
\end{aligned}
$$

From $(48)_{1}$, we have that $\left(u_{\varepsilon l m}^{\prime}\right)$ is bounded in $L_{\text {loc }}^{\infty}(0, \infty$; $\left.H^{1 / 2}\left(\Gamma_{1}\right)\right)$. Thus, estimate $(49)_{3}$ and the compactness embedding of $H^{1 / 2}\left(\Gamma_{1}\right)$ in $L^{2}\left(\Gamma_{1}\right)$, give $u_{\varepsilon l m}^{\prime} \rightarrow u_{\varepsilon l}^{\prime}$ in $L_{\text {loc }}^{2}(0, \infty$; $\left.L^{2}\left(\Gamma_{1}\right)\right)$. From this, property (iii) and Lemma 7 yield

$$
h_{l}\left(\cdot, u_{\varepsilon l m}^{\prime}\right) \longrightarrow h_{l}\left(\cdot, u_{\varepsilon l}^{\prime}\right) \quad \text { in } L_{\mathrm{loc}}^{2}\left(0, \infty ; L^{2}\left(\Gamma_{1}\right)\right)
$$

3.1.4. Passage to the Limit as $m$. Convergences (49), (51), and (53) permit us to pass to the limits in approximate equations $(A P)$, as $m \rightarrow \infty$. Thus, this fact and the density of $V_{m}^{l}$ in $V$, give

$$
\begin{aligned}
& \int_{0}^{T}\left((K+\varepsilon) u_{\varepsilon l}^{\prime \prime}(t), v\right) \psi(t) d t+\int_{0}^{T}\left(\left(u_{\varepsilon l}(t), v\right)\right) \psi(t) d t \\
& \quad+\int_{0}^{T} \int_{\Gamma_{1}} h_{l}\left(\cdot, u_{\varepsilon l}^{\prime}(t)\right) v \psi(t) d \Gamma d t \\
& +\sum_{i=1}^{n} \int_{0}^{T}\left(b_{i} \frac{\partial u_{\varepsilon l}^{\prime}}{\partial x_{i}}(t), v\right) \psi(t) d t \\
& +\int_{0}^{T}\left(\left(\left|u_{\varepsilon l}(t)\right|^{\sigma} u_{\varepsilon}(t)\right), v\right) \psi(t)=0,
\end{aligned}
$$$$
\forall \psi \in \mathscr{D}(0, T), \quad \forall v \in V .
$$ 
Now, if $\psi \in \mathscr{D}(0, T)$ and $v \in \mathscr{D}(\Omega)$ we obtain using the regularity of $u_{\varepsilon l}$ (given by (49)), that

$$
\begin{aligned}
& (K+\varepsilon) u_{\varepsilon l}^{\prime \prime}-\Delta u_{\varepsilon l}+\sum_{i=1}^{n} b_{i} \frac{\partial u_{\varepsilon l}^{\prime}}{\partial x_{i}} \\
& +\left|u_{\varepsilon l}\right|^{\sigma} u_{\varepsilon l}=0 \quad \text { in } L_{\text {loc }}^{\infty}\left(0, \infty ; L^{2}(\Omega)\right) .
\end{aligned}
$$

From (49) $)_{1}$ and (55) we have $u_{\varepsilon l} \in L^{\infty}(0, T ; V)$ and $\Delta u_{\varepsilon l} \in$ $L^{\infty}\left(0, T ; L^{2}(\Omega)\right)$, respectively. Thus, $\partial u_{\varepsilon l} / \partial \nu \in L^{\infty}(0, T$; $\left.H^{-1 / 2}\left(\Gamma_{1}\right)\right)$. (compare to Lions [19] and Medeiros and Milla Miranda [21]). Multiplying both sides of (55) by $v \psi$, with $v \in V$ and $\psi \in \mathscr{D}(0, T)$, and integrating over $\Omega \times[0, T[$, then the preceding regularity, $\partial u_{\varepsilon l} / \partial \nu$, gives

$$
\begin{aligned}
& \int_{0}^{T}\left((K+\varepsilon) u_{\varepsilon l}^{\prime \prime}, v\right) \psi d t+\int_{0}^{T}\left(\left(u_{\varepsilon l}, v\right)\right) \psi d t \\
& -\int_{0}^{T}\left\langle\frac{\partial u_{\varepsilon l}}{\partial v}, v\right\rangle \psi d t+\int_{0}^{T}\left(\left|u_{\varepsilon l}\right|^{\sigma} u_{\varepsilon l}, v\right) \psi d t \\
& +\sum_{i=1}^{n} \int_{0}^{T}\left(b_{i} \frac{\partial u_{\varepsilon l}^{\prime}}{\partial x_{i}}, v\right) \psi d t=0,
\end{aligned}
$$

where $\langle\cdot, \cdot\rangle$ denotes the duality paring between $H^{-1 / 2}\left(\Gamma_{1}\right)$ and $H^{1 / 2}\left(\Gamma_{1}\right)$.

Comparing (54) and (56) and using the Lipschitz property of $h_{l}$, we obtain

$$
\frac{\partial u_{\varepsilon l}}{\partial \nu}+h_{l}\left(\cdot, u_{\varepsilon l}^{\prime}\right)=0 \quad \text { in } L_{\text {loc }}^{2}\left(0, \infty ; L^{2}\left(\Gamma_{1}\right)\right)
$$

3.1.5. Passage to the Limit in $\varepsilon \rightarrow 0$ and $l \rightarrow \infty$. As estimates (36) and (49) are independent of $l, m$, and $\varepsilon$ we obtain a subsequence of $\left(u_{\varepsilon l}\right)$, which still denoted by $\left(u_{\varepsilon l}\right)$, and a function $u_{l}$ such that all convergences (49) and (52) are valid. These convergences will be denoted by $(49)_{\varepsilon},(51)_{\varepsilon}$, and $(52)_{\varepsilon}$, respectively. These results imply that there exists a function $u_{l}$ belonging to class (49) and it is a solution of equation

$$
\begin{aligned}
K u_{l}^{\prime \prime}-\Delta u_{l}+\sum_{i=1}^{n} b_{i} \frac{\partial u_{l}^{\prime}}{\partial x_{i}} \\
+\left|u_{l}\right|^{\sigma} u_{l}=0 \quad \text { in } L_{\text {loc }}^{\infty}\left(0, \infty ; L^{2}(\Omega)\right), \\
\frac{\partial u_{l}}{\partial v}+h_{l}\left(\cdot, u_{l}^{\prime}\right)=0 \quad \text { in } L_{\text {loc }}^{2}\left(0, \infty ; L^{2}\left(\Gamma_{1}\right)\right) .
\end{aligned}
$$

Denoting these convergence in $l$ by $(49)_{l},(51)_{l}$, and $(52)_{l}$, respectively, then the convergence (49), gives us $u_{l} \rightarrow u$ weak star in $L^{\infty}(0, \infty ; V)$. From this and (55), $\Delta u_{l} \rightarrow \Delta u$ weak star in $L_{\text {loc }}^{\infty}\left(0, \infty ; L^{2}(\Omega)\right)$. Then

$$
\frac{\partial u_{l}}{\partial v} \longrightarrow \frac{\partial u}{\partial v} \text { weak star in } L_{\text {loc }}^{\infty}\left(0, \infty ; H^{-1 / 2}\left(\Gamma_{1}\right)\right) \text {. }
$$

Moreover, convergence (49) $)_{l}$ furnishes $u_{l}^{\prime} \rightarrow u^{\prime}$ in $L_{\text {loc }}^{2}(0$, $\left.\infty ; L^{2}\left(\Gamma_{1}\right)\right)$. Now, we fix $T>0$. The preceding convergence implies

$$
\left.u_{l}^{\prime}(x, t) \longrightarrow u^{\prime}(x, t) \quad \text { a.e in } \Sigma_{1}=\Gamma_{1} \times\right] 0, T[.
$$

Fixing $(x, t) \in \Sigma_{1}$, then by (61) the set $\left\{u_{l}^{\prime}(x, t): l \in \mathbb{N}\right\}$ is bounded. Part (iv) of Lemma 7 says that $\left(h_{l}\right)$ converges to $h$ uniformly in bounded sets of $\mathbb{R}$, a.e. $x$ in $\Gamma_{1}$. These two results and (61) give

$$
\begin{aligned}
& h_{l}\left(x, u_{l}^{\prime}(x, t)\right) \\
& \left.\quad \longrightarrow h\left(x, u^{\prime}(x, t)\right) \quad \text { a.e in } \Sigma_{1}=\Gamma_{1} \times\right] 0, T[.
\end{aligned}
$$

On the other hand, by (58) and (59), we obtain

$$
\begin{aligned}
\int_{\Gamma_{1}} h_{l}\left(\cdot, u_{l}^{\prime}(t)\right) u_{l}^{\prime}(t) d \Gamma \\
=-\left(K(t) u_{l}^{\prime \prime}(t), u_{l}^{\prime}(t)\right)-\frac{1}{2} \frac{d}{d t}\left\|u_{l}(t)\right\|^{2} \\
\quad-\sum_{i=1}^{n}\left(b_{i} \frac{\partial u_{l}^{\prime}(t)}{\partial x_{i}}, u_{l}^{\prime}(t)\right)-\left(\left|u_{l}(t)\right|^{\sigma} u_{l}(t), u_{l}^{\prime}(t)\right) .
\end{aligned}
$$

By familiar inequalities,

$$
\left|\sum_{i=1}^{n}\left(b_{i} \frac{\partial u_{l}^{\prime}(t)}{\partial x_{i}}, u_{l}^{\prime}(t)\right)\right| \leq C\left[\left\|u_{l}^{\prime}(t)\right\|^{2}+\left|u_{l}^{\prime}(t)\right|^{2}\right],
$$

and from embedding (17),

$$
\begin{aligned}
& \left|\left(\left|u_{l}(t)\right|^{\sigma} u_{l}(t), u_{l}^{\prime}(t)\right)\right| \\
& \quad \leq C\left[\left\|u_{l}(t)\right\|^{2(\sigma+2)}+\left|u_{l}^{\prime}(t)\right|^{2}\right],
\end{aligned}
$$

where $C>0$ is a constant independent of $l$ and $t \in[0, T]$.

As

$$
\begin{aligned}
\left(K(t) u_{l}^{\prime \prime}(t), u_{l}^{\prime}(t)\right)= & \frac{1}{2} \frac{d}{d t}\left(K(t) u_{l}^{\prime}(t), u_{l}^{\prime}(t)\right) \\
& -\frac{1}{2}\left(K^{\prime}(t) u_{l}^{\prime}(t), u_{l}^{\prime}(t)\right),
\end{aligned}
$$

integrating the inequality above from 0 to $T$, and using the hypothesis (H5) and (H6), we find

$$
\begin{aligned}
\int_{0}^{T}\left(K(t) u_{l}^{\prime \prime}(t), u_{l}^{\prime}(t)\right) d t \\
=\frac{1}{2}\left[\left(K(T) u_{l}^{\prime}(T), u_{l}^{\prime}(T)\right)-\left(K(0) u_{l}^{1}, u_{l}^{1}\right)\right] \\
\quad-\frac{1}{2} \int_{0}^{T}\left(K^{\prime}(t) u_{l}^{\prime}(t), u_{l}^{\prime}(t)\right) d t \\
\leq\|K\|_{\infty}\left[\left|u_{l}^{\prime}(T)\right|^{2}+\left|u_{l}^{1}\right|^{2}\right] \\
\quad+\int_{0}^{T}\left[\delta\left|u_{l}^{\prime}(s)\right|^{2}+C(\delta)\left(K(s), u_{l}^{\prime 2}(s)\right)\right] d s \\
\leq\|K\|_{\infty}\left[\left|u_{l}^{\prime}(T)\right|^{2}+\left|u_{l}^{1}\right|^{2}\right] \\
\quad+C\left(\delta,\|K\|_{\infty}\right) \int_{0}^{T}\left|u_{l}^{\prime}(t)\right|^{2} d t .
\end{aligned}
$$


Note that $u_{l} \in C^{0}([0, T] ; V), u_{l}^{\prime} \in C^{0}\left([0, T] ; L^{2}(\Omega)\right)$ and that $\left(u_{l}(T)\right)$ and $\left(u_{l}^{\prime}(T)\right)$ are bounded in $V$ and in $L^{2}(\Omega)$, respectively. Taking into account the preceding considerations, estimates (49) $)_{l}$ and convergence (36) $)_{l}$ in (63), we obtain

$$
\begin{array}{r}
\int_{0}^{T} \int_{\Gamma_{1}} h_{l}\left(\cdot, u_{l}^{\prime}(t)\right) u_{l}^{\prime}(t) d \Gamma d t \leq C, \\
\forall l \geq l_{0}, \quad \forall t \in[0, T],
\end{array}
$$

where $C>0$ is a constant independent of $l \geq l_{0}$ and $t \in[0, T]$. Note that $h_{l}\left(\cdot, u_{l}^{\prime}(t)\right) u_{l}^{\prime}(t) \geq 0$.

From (62), (68), Strauss' approximations, Lemma 9 and from a diagonal process, we get

$$
h_{l}\left(\cdot, u_{l}^{\prime}\right) \longrightarrow h\left(\cdot, u^{\prime}\right) \quad \text { in } L_{\text {loc }}^{1}\left(0, \infty ; L^{1}\left(\Gamma_{1}\right)\right) \text {. }
$$

Convergences (60) and (69) imply $\partial u_{l} / \partial v \rightarrow \partial u / \partial v$ in $\mathscr{D}^{\prime}\left(\Gamma_{1} \times\right] 0, \infty[)$ and $h_{l}\left(\cdot, u_{l}^{\prime}\right) \rightarrow h\left(\cdot, u^{\prime}\right)$ in $\mathscr{D}^{\prime}\left(\Gamma_{1} \times\right] 0, \infty[)$. Now we take the limit in (59). Moreover the last two convergences and the regularity of $h\left(\cdot, u^{\prime}\right)$ imply $\partial u / \partial v+$ $h\left(\cdot, u^{\prime}\right)=0$ in $L_{\text {loc }}^{1}\left(0, \infty ; L^{1}\left(\Gamma_{1}\right)\right)$, which shows that $u$ satisfies

$$
\begin{array}{r}
K u^{\prime \prime}-\Delta u+\sum_{i=1}^{n} b_{i} \frac{\partial u^{\prime}}{\partial x_{i}}+|u|^{\sigma} u=0 \\
\text { in } L_{\text {loc }}^{\infty}\left(0, \infty ; L^{2}(\Omega)\right) .
\end{array}
$$

Hence, the result is done as in (58).

The verification of the initial conditions follows by convergence (49) .

Remark 10. If $|h(x, s)| \leq h_{1}|s|$ a.e. $x \in \Gamma$, then the sequence $h_{l}$, which converges to $h$, satisfies $\left|h_{l}(x, s)\right| \leq(3 / 2) h_{1}|s|$ (see Lourêdo and Miranda [10]). In these conditions, the solution is unique and $\partial u / \partial v+h\left(\cdot, u^{\prime}\right)=0$ in $L_{\text {loc }}^{2}\left(0, \infty ; L^{2}\left(\Gamma_{1}\right)\right)$. Consequently, $u \in L^{\infty}\left(0, \infty, V \cap H^{3 / 2}(\Omega)\right)$.

The proof of Corollary 2 follows from Remark 10 and from regularity of elliptic problems (see Lions and Magenes [22]).

\section{Asymptotic Behavior}

In this section, by applying Nakao's method (see [23]), we will prove the uniform stabilization of the energy associated with the solution of the Problem $(P)$.

Proof of Theorem 6. First, we prove the inequality (15) for the approximate energy $E_{l}(t)$ given by

$$
\begin{aligned}
E_{l}(t)=\frac{1}{2}\left\{K^{1 / 2}(t)\left|u_{l}^{\prime}(t)\right|^{2}+\left\|u_{l}(t)\right\|^{2}\right. & \\
& \left.+\frac{2}{\sigma+2} \int_{\Omega}\left|u_{l}(t)\right|^{\sigma+2} d x\right\} \quad \text { for } t \geq 1,
\end{aligned}
$$

and Theorem 6 will follow by taking the lim inf in $l$.

Taking the scalar product of $L^{2}(\Omega)$ in both sides of

$$
\begin{array}{r}
K u_{l}^{\prime \prime}-\Delta u_{l}+\sum_{j=1}^{n} b_{j} \frac{\partial u_{l}^{\prime}}{\partial x_{j}}+\left|u_{l}\right|^{\sigma} u_{l}=0 \\
\quad \text { in } L_{\text {loc }}^{\infty}\left(0, \infty ; L^{2}(\Omega)\right)
\end{array}
$$

with $u_{l}^{\prime}$, we find

$$
\begin{aligned}
\frac{d}{d t} E_{l}(t)= & \frac{1}{2}\left(\frac{\partial K(t)}{\partial t} u_{l}^{\prime}(t), u_{l}^{\prime}(t)\right) \\
& -\left(h_{l}\left(\cdot, u_{l}^{\prime}(t)\right), u_{l}^{\prime}(t)\right)_{\Gamma_{1}} \\
& +\frac{1}{2} \int_{\Omega} \operatorname{div} b(t)\left|u_{l}^{\prime}(t)\right|^{2} d x \\
& -\frac{1}{2} \int_{\Gamma_{1}} b(t) \cdot v\left|u_{l}(t)\right|^{2} d x .
\end{aligned}
$$

Using (73) and the hypotheses (A2), (H3), (H8), and (H2), we obtain

$$
\frac{d}{d t} E_{l}(t) \leq-\frac{b_{0}}{4}\left|u_{l}^{\prime}(t)\right|^{2}+\left(\frac{\|b\|_{\infty}}{2}-h_{0}\right)\left|u_{l}^{\prime}(t)\right|_{\Gamma_{1}}^{2} \leq 0 .
$$

Note that $E_{l}(t)$ is decreasing. From (73), the hypotheses $(H 1)$, $(H 2)$, and Remark 10 , we have that

$$
\begin{aligned}
\frac{d}{d t} E_{l}(t) \leq & \frac{b_{0}}{2}\left|u_{l}^{\prime}(t)\right|^{2}+d_{1}\left|u_{l}^{\prime}(t)\right|_{\Gamma_{1}}^{2}+\frac{\delta_{0}}{2}\left|u_{l}^{\prime}(t)\right|^{2} \\
& +\frac{\|b\|_{\infty}}{2}\left|u_{l}^{\prime}(t)\right|_{\Gamma_{1}}^{2} .
\end{aligned}
$$

Thus,

$$
\frac{d}{d t} E_{l}(t) \leq N_{1}\left|u_{l}^{\prime}(t)\right|^{2}+N_{2}\left|u_{l}^{\prime}(t)\right|_{\Gamma_{1}}^{2},
$$

where $N_{1}=\left(b_{0} / 2+\delta_{0} / 2\right)>0$ and $N_{2}=d_{1}+\left(\|b\|_{\infty} / 2\right)>0$. Integrating (76) from $t$ to $t+1$, we obtain

$$
\begin{aligned}
E_{l}(t+1)-E_{l}(t) \leq & N_{1} \int_{t}^{t+1}\left|u_{l}^{\prime}(s)\right|^{2} d s \\
& +N_{2} \int_{t}^{t+1}\left|u_{l}^{\prime}(s)\right|_{\Gamma_{1}}^{2} d s .
\end{aligned}
$$

Furthermore, taking the scalar product in both sides of (72) with $u_{l}$, we find

$$
\begin{aligned}
& \left(K(t) u_{l}^{\prime \prime}(t), u_{l}(t)\right)+\left\|u_{l}(t)\right\|^{2}+\left(b \cdot \nabla u_{l}^{\prime}(t), u_{l}(t)\right) \\
& \quad+\left(h_{l}\left(\cdot, u_{l}^{\prime}(t)\right), u_{l}(t)\right)_{\Gamma_{1}}+\left(\left|u_{l}(t)\right|^{\sigma} u_{l}(t), u_{l}(t)\right)=0 .
\end{aligned}
$$

As

$$
\begin{aligned}
\left(K(t) u_{l}^{\prime \prime}(t), u_{l}(t)\right)= & \frac{d}{d t}\left(K(t) u_{l}^{\prime}(t), u_{l}(t)\right) \\
& -\left(K^{\prime}(t) u_{l}^{\prime}(t), u_{l}(t)\right) \\
& -\left(K(t) u_{l}^{\prime}(t), u_{l}^{\prime}(t)\right),
\end{aligned}
$$


then

$$
\begin{aligned}
\left\|u_{l}(t)\right\|^{2} & +\left|u_{l}(t)\right|_{L^{\sigma+2}(\Omega)}^{\sigma+2} \\
= & -\left(b \cdot \nabla u_{l}^{\prime}(t), u_{l}(t)\right)-\left(h_{l}\left(\cdot, u_{l}^{\prime}(t)\right), u_{l}(t)\right)_{\Gamma_{1}} \\
& +\left(K^{\prime}(t) u_{l}^{\prime}(t), u_{l}(t)\right)+\left(K(t) u_{l}^{\prime}(t), u_{l}^{\prime}(t)\right) \\
& -\frac{d}{d t}\left(K(t) u_{l}^{\prime}(t), u_{l}(t)\right) .
\end{aligned}
$$

Now note that

$$
\left(b \cdot \nabla u_{l}^{\prime}(t), u_{l}(t)\right)=\int_{\Omega} \sum_{j=1}^{n} b_{j}(t) \frac{\partial u_{l}^{\prime}}{\partial x_{j}}(t) u_{l}(t) d x
$$

and by Gauss' formula, we have

$$
\begin{aligned}
\frac{\partial}{\partial x_{j}}\left(b_{j}(t) u_{l}(t) u_{l}^{\prime}(t)\right)= & b_{j}(t) u_{l}(t) \frac{\partial u_{l}^{\prime}}{\partial x_{j}}(t) \\
& +\frac{\partial b_{j}(t)}{\partial x_{j}} u_{l}(t) u_{l}^{\prime}(t) \\
& +b_{j}(t) \frac{\partial u_{l}}{\partial x_{j}}(t) u_{l}^{\prime}(t) .
\end{aligned}
$$

Therefore,

$$
\begin{aligned}
\sum_{j=1}^{n} \int_{\Omega} b_{j}(t) u_{l}(t) \frac{\partial u_{l}^{\prime}(t)}{\partial x_{j}} d x= & \int_{\Gamma} b(t) \cdot v u_{l}(t) u_{l}^{\prime}(t) d \Gamma \\
& -\int_{\Omega}(\operatorname{div} b(t)) u_{l}(t) u_{l}^{\prime}(t) d x \\
& -\int_{\Omega} b(t) \cdot \nabla u_{l}(t) u_{l}^{\prime}(t) d x .
\end{aligned}
$$

Hence,

$$
\begin{aligned}
\left(b \cdot \nabla u_{l}^{\prime}(t), u_{l}(t)\right)= & \int_{\Gamma_{1}} b(t) v u_{l}(t) u_{l}^{\prime}(t) d \Gamma \\
& -\int_{\Omega}(\operatorname{div} b(t)) u_{l}(t) u_{l}^{\prime}(t) d x \\
& -\int_{\Omega} b(t) \cdot \nabla u_{l}(t) u_{l}^{\prime}(t) d x .
\end{aligned}
$$

Substituting (84) in (80), we obtain

$$
\begin{aligned}
& \left\|u_{l}(t)\right\|^{2}+\left|u_{l}(t)\right|_{L^{\sigma+2}(\Omega)}^{\sigma+2} \\
& =-\int_{\Gamma_{1}} b(t) \cdot v u_{l}(t) u_{l}^{\prime}(t) d \Gamma+\int_{\Omega}(\operatorname{div} b(t)) u_{l}(t) u_{l}^{\prime}(t) d x \\
& \quad+\int_{\Omega} b(t) \cdot \nabla u_{l}(t) u_{l}^{\prime}(t) d x-\left(h_{l}\left(\cdot, u_{l}^{\prime}(t)\right), u_{l}(t)\right)_{L^{2}\left(\Gamma_{1}\right)} \\
& \quad+\left(K^{\prime}(t) u_{l}^{\prime}(t), u_{l}(t)\right)+\left(K(t) u_{l}^{\prime}(t), u_{l}^{\prime}(t)\right) \\
& \quad-\frac{d}{d t}\left(K(t) u_{l}^{\prime}(t), u_{l}(t)\right) .
\end{aligned}
$$

Integrating (85) from $t_{1}$ to $t_{2}$ yields

$$
\begin{aligned}
& \int_{t_{1}}^{t_{2}}\left(\left\|u_{l}(t)\right\|^{2}+\left|u_{l}(t)\right|_{L^{\sigma+2}(\Omega)}^{\sigma+2}\right) d t \\
& \leq\|b\|_{\infty} \int_{t_{1}}^{t_{2}}\left|u_{l}(t)\right|_{\Gamma_{1}}\left|u_{l}^{\prime}(t)\right|_{\Gamma_{1}} d t+\delta_{0} \int_{t_{1}}^{t_{2}}\left|u_{l}(t)\right|\left|u_{l}^{\prime}(t)\right| d t \\
& +\|b\|_{\infty} \int_{t_{1}}^{t_{2}}\left|\nabla u_{l}(t)\right|\left|u_{l}^{\prime}(t)\right| d t \\
& +\int_{t_{1}}^{t_{2}}\left|h_{l}\left(\cdot, u_{l}^{\prime}(t)\right)\right|_{\Gamma_{1}}\left|u_{l}(t)\right|_{\Gamma_{1}} d t \\
& +\int_{t_{1}}^{t_{2}} \frac{b_{0}}{2}\left|u_{l}^{\prime}(t)\right|\left|u_{l}(t)\right| d t+\|K\|_{\infty} \int_{t_{1}}^{t_{2}}\left|u_{l}^{\prime}(t)\right|^{2} d t \\
& \quad-\left[\left(K\left(t_{2}\right) u_{l}^{\prime}\left(t_{2}\right), u_{l}\left(t_{2}\right)\right)-\left(K\left(t_{1}\right) u_{l}^{\prime}\left(t_{1}\right), u_{l}\left(t_{1}\right)\right)\right] .
\end{aligned}
$$

Using the embedding of $V$ in $L^{2}(\Omega)$ and $V$ in $L^{2}\left(\Gamma_{1}\right)$ (see (12)), it follows that

(i) $\|b\|_{\infty} \int_{t_{1}}^{t_{2}}\left|u_{l}(t)\right|_{\Gamma_{1}}\left|u_{l}^{\prime}(t)\right|_{\Gamma_{1}} d t \leq 32\|b\|_{\infty}^{2} k_{1}^{2} \int_{t_{1}}^{t_{2}}\left|u_{l}^{\prime}(t)\right|_{\Gamma_{1}}^{2} d t$ $+(1 / 32) \int_{t_{1}}^{t_{2}}\left\|u_{l}(t)\right\|^{2} d t$

(ii) $\delta_{0} \int_{t_{1}}^{t_{2}}\left|u_{l}(t)\right|\left|u_{l}^{\prime}(t)\right| d t \leq 32\left(1 / \lambda_{1}\right) \int_{t_{1}}^{t_{2}}\left|u_{l}^{\prime}(t)\right|^{2} d t+(1 /$ 32) $\int_{t_{1}}^{t_{2}}\left\|u_{l}(t)\right\|^{2} d t$

(iii) $\|b\|_{\infty} \int_{t_{1}}^{t_{2}}\left|\nabla u_{l}(t)\left\|u_{l}^{\prime}(t) \mid d t \leq\left(\|b\|_{\infty}^{2} / 2\right) \int_{t_{1}}^{t_{2}}\right\| u_{l}(t) \|^{2} d t+\right.$ $(1 / 2) \int_{t_{1}}^{t_{2}}\left|u_{l}^{\prime}(t)\right|^{2} d t$

(iv) $\int_{t_{1}}^{t_{2}}\left|h_{l}\left(\cdot, u_{l}^{\prime}(t)\right)\right|_{\Gamma_{1}}\left|u_{l}(t)\right|_{\Gamma_{1}} d t \leq(3 / 2) h_{1} k_{1} \int_{t_{1}}^{t_{2}}\left|u_{l}^{\prime}(t)\right|_{\Gamma_{1}}$. $\left|u_{l}(t)\right|_{\Gamma_{1}} d t \leq\left((3 / 2) h_{1} k_{1}\right)^{2}\left(\lambda_{1}^{1 / 2}\right)^{2} 16 \int_{t_{1}}^{t_{2}}\left|u_{l}^{\prime}(t)\right|_{\Gamma_{1}}^{2} d t+$ $(1 / 32) \int_{t_{1}}^{t_{2}}\left\|u_{l}(t)\right\|^{2} d t$

(v) $\int_{t_{1}}^{t_{2}}\left(b_{0} / 2\right)\left|u_{l}^{\prime}(t)\right|\left|u_{l}(t)\right| d t \leq 32\left(b_{0} / 2\right)^{2}\left(1 / \lambda_{1}\right) \int_{t_{1}}^{t_{2}}\left|u_{l}^{\prime}(t)\right|^{2}$ $+(1 / 32) \int_{t_{1}}^{t_{2}}\left\|u_{l}(t)\right\|^{2} d t$

(vi) $\mid\left(K\left(t_{2}\right) u_{l}^{\prime}\left(t_{2}\right), u_{l}\left(t_{2}\right)\right)-\left(K\left(t_{1}\right) u_{l}^{\prime}\left(t_{1}\right), u_{l}\left(t_{1}\right) \mid \leq\|K\|_{\infty}\right.$. $\left[\left|u_{l}^{\prime}\left(t_{2}\right)\right|\left|u_{l}\left(t_{2}\right)\right|+\left|u_{l}^{\prime}\left(t_{1}\right)\right|\left|u_{l}\left(t_{1}\right)\right|\right]$.

Note that

$$
\left|u_{l}\left(t_{2}\right)\right| \leq\left(\frac{1}{\sqrt{\lambda_{1}}}\right)\left\|u_{l}(t)\right\| \leq\left(\frac{1}{\sqrt{\lambda_{1}}}\right) E_{l}^{1 / 2}\left(t_{2}\right)
$$

since $E_{l}(t)$ is decreasing and $t \leq t_{1}, t_{2} \leq t+1$. Analogously, we obtain

$$
\left|u_{l}\left(t_{1}\right)\right| \leq\left(\frac{1}{\sqrt{\lambda_{1}}}\right) E_{l}^{1 / 2}\left(t_{1}\right)
$$


Using (87) and (88) in (vi) we find

$$
\begin{aligned}
& \left|\left(K\left(t_{2}\right) u_{l}^{\prime}\left(t_{2}\right), u_{l}\left(t_{2}\right)\right)-\left(K\left(t_{1}\right) u_{l}^{\prime}\left(t_{1}\right), u_{l}\left(t_{1}\right)\right)\right| \\
& \quad \leq\|K\|_{\infty}\left(\frac{1}{\lambda_{1}^{1 / 2}}\right) \sup _{t_{1} \leq s \leq t_{2}} E_{l}^{1 / 2}(s)\left[\left|u_{l}^{\prime}\left(t_{2}\right)\right|+\left|u_{l}^{\prime}\left(t_{1}\right)\right|\right] .
\end{aligned}
$$

Substituting (i)-(vi) and (89) in (86), we obtain

$$
\begin{aligned}
\int_{t_{1}}^{t_{2}}[ & \left.\left\|u_{l}(t)\right\|^{2}+\left|u_{l}(t)\right|_{L^{\sigma+2}(\Omega)}^{\sigma+2}\right] d t \\
\leq & \left(32+\|b\|_{\infty} \frac{1}{\lambda_{1}}\right) \int_{t_{1}}^{t_{2}}\left\|u_{l}(t)\right\|^{2} d t \\
& +\left(32\left(\frac{1}{\lambda_{1}}\right)^{2}+8 b_{0}^{2}\left(\frac{1}{\lambda_{1}}\right)^{2}\right) \int_{t_{1}}^{t_{2}}\left|u_{l}^{\prime}(t)\right|^{2} d t \\
& +\left(32\|b\|_{\infty}^{2} k_{1}^{2}+18\left(h_{1} k_{1}\right)^{2} \lambda_{1}+\frac{1}{2}\right) \int_{t_{1}}^{t_{2}}\left|u_{l}^{\prime}(t)\right|_{\Gamma_{1}}^{2} d t \\
& +\|K\|_{\infty}\left(\frac{1}{\sqrt{\lambda_{1}}}\right) \sup _{t_{1} \leq s \leq t_{2}} E_{l}^{1 / 2}(s)\left[\left|u_{l}^{\prime}\left(t_{2}\right)\right|+\left|u_{l}^{\prime}\left(t_{1}\right)\right|\right] .
\end{aligned}
$$

It follows from (74) that

$$
\begin{aligned}
E_{l}(t+1)-E_{l}(t) \leq & -N_{3} \int_{t}^{t+1}\left|u_{l}^{\prime}(s)\right|^{2} d s \\
& -N_{4} \int_{t}^{t+1}\left|u_{l}^{\prime}(s)\right|_{\Gamma_{1}} d s,
\end{aligned}
$$

where $N_{3}=b_{0}>0$ and $N_{4}=h_{0}-\|b\|_{\infty} / 2>0$. Hence

$$
\begin{aligned}
D_{l}^{2}(t) & =E_{l}(t)-E_{l}(t+1) \\
& \geq N_{3} \int_{t}^{t+1}\left|u_{l}^{\prime}(s)\right|^{2} d s+N_{4} \int_{t}^{t+1}\left|u_{l}^{\prime}(t)\right|_{\Gamma_{1}}^{2} d s \\
& \geq d_{1}\left[\int_{t}^{t+1}\left|u_{l}^{\prime}(s)\right|^{2} d s+\int_{t}^{t+1}\left|u_{l}^{\prime}(t)\right|_{\Gamma_{1}}^{2} d s\right],
\end{aligned}
$$

and from (93), we get

$$
\begin{gathered}
\int_{t}^{t+1 / 4}\left|u_{l}^{\prime}(s)\right|_{\Gamma_{1}}^{2} d s \leq \int_{t}^{t+1}\left|u_{l}^{\prime}(s)\right|_{\Gamma_{1}}^{2} d s \leq \frac{D_{l}^{2}(t)}{d_{1}}, \\
\int_{t+3 / 4}^{t+1}\left|u_{l}^{\prime}(s)\right|_{\Gamma_{1}}^{2} d s \leq \int_{t}^{t+1}\left|u_{l}^{\prime}(s)\right|_{\Gamma_{1}}^{2} d s \leq \frac{D_{l}^{2}(t)}{d_{1}} .
\end{gathered}
$$

By the Mean Value Theorem, there are $t_{1} \in(t, t+1 / 4)$ and $t_{2} \in(t+3 / 4, t+1)$, such that

$$
\begin{aligned}
& \frac{1}{4}\left|u_{l}^{\prime}\left(t_{1}\right)\right|^{2}=\int_{t}^{t+1 / 4}\left|u_{l}^{\prime}(s)\right|^{2} d s \leq \frac{D_{l}^{2}(t)}{d_{1}}, \\
& \frac{1}{4}\left|u_{l}^{\prime}\left(t_{2}\right)\right|^{2}=\int_{t+3 / 4}^{t+1}\left|u_{l}^{\prime}(s)\right|^{2} d s \leq \frac{D_{l}^{2}(t)}{d_{1}} .
\end{aligned}
$$

From (95), we can write

$$
\left|u_{l}^{\prime}\left(t_{1}\right)\right|+\left|u_{l}^{\prime}\left(t_{2}\right)\right| \leq \frac{2 D_{l}(t)}{\sqrt{d_{1}}} .
$$

Now from (93) we have

$$
\begin{aligned}
N_{5} \int_{t_{1}}^{t_{2}}\left|u_{l}^{\prime}(s)\right|_{\Gamma_{1}}^{2} d s & \leq N_{5} \int_{t}^{t+1}\left|u_{l}^{\prime}(s)\right|_{\Gamma_{1}}^{2} d s \\
& \leq \frac{N_{5}}{d_{1}} D_{l}^{2}(t),
\end{aligned}
$$

where $N_{5}=32\|b\|_{\infty}+18\left(h_{1} k_{1}\right)^{2}$.

Analogously we obtain

$$
N_{6} \int_{t_{1}}^{t_{2}}\left|u_{l}^{\prime}(s)\right|^{2} d s \leq N_{6} \int_{t}^{t+1}\left|u_{l}^{\prime}(s)\right|^{2} d s \leq \frac{N_{6}}{d_{1}} D_{l}^{2}(t),
$$

where $N_{6}=32\left(1 / \lambda_{1}\right)+8 b_{0}^{2}\left(1 / \lambda_{1}\right)$.

Substituting (96), (97), and (98) in (90) and considering

$$
N_{7}=\frac{9}{32}+\left(\frac{1}{\lambda_{1}}\right)\|b\|_{\infty},
$$

we get

$$
\begin{aligned}
& \int_{t_{1}}^{t_{2}}\left[\left\|u_{l}(t)\right\|^{2}+\left|u_{l}(t)\right|_{L^{\sigma+2}(\Omega)}^{\sigma+2}\right] d t \\
& \leq N_{7} \int_{t_{1}}^{t_{2}}\left\|u_{l}(t)\right\|^{2} d t+N_{5} \int_{t_{1}}^{t_{2}}\left|u_{l}^{\prime}(t)\right|^{2} d t \\
&+N_{6} \int_{t_{1}}^{t_{2}}\left|u_{l}^{\prime}(t)\right|_{\Gamma_{1}}^{2} d t \\
&+\|K\|_{\infty}\left(\frac{1}{\sqrt{\lambda_{1}}}\right) \sup _{t_{1} \leq s \leq t_{2}} E_{l}^{1 / 2}\left[\left|u_{l}^{\prime}\left(t_{2}\right)\right|+\left|u_{l}^{\prime}\left(t_{1}\right)\right|\right] \\
& \leq N_{7} \int_{t_{1}}^{t_{2}}\left\|u_{l}(t)\right\|^{2} d t+\frac{N_{5}}{d_{1}} D_{l}^{2}(t)+\frac{N_{6}}{d_{1}} D_{l}^{2}(t) \\
&+\frac{2\|K\|_{\infty}\left(1 / \sqrt{\lambda_{1}}\right)}{\sqrt{d_{1}}} \sup _{t_{1} \leq s \leq t_{2}} E_{l}^{1 / 2} D_{l}(t) \\
& \leq N_{7} \int_{t_{1}}^{t_{2}}\left\|u_{l}(t)\right\|^{2} d t+\left(\frac{N_{5}}{d_{1}}+\frac{N_{6}}{d_{1}}\right) D_{l}^{2}(t) \\
&+\frac{2\|K\|_{\infty}\left(1 / \sqrt{\lambda_{1}}\right)}{\sqrt{d_{1}}} D_{l}(t) E_{l}^{1 / 2}(t) .
\end{aligned}
$$


As $D_{l}^{2}(t)=E_{l}(t)-E_{l}(t+1) \leq E_{l}(t)$ then $D_{l}(t) \leq E_{l}^{1 / 2}(t)$, and substituting this inequality in (100), we obtain

$$
\begin{aligned}
\int_{t_{1}}^{t_{2}}\left(\left\|u_{l}(t)\right\|^{2}+\left|u_{l}(t)\right|_{L^{\sigma+2}(\Omega)}^{\sigma+2}\right) d t \\
\leq N_{7} \int_{t_{1}}^{t_{2}}\left\|u_{l}(t)\right\|^{2} d t+\left(\frac{N_{5}+N_{6}}{d_{1}}\right) D_{l}^{2}(t) \\
\quad+\frac{2\|K\|_{\infty}\left(1 / \sqrt{\lambda_{1}}\right)}{\sqrt{d_{1}}} D_{l}(t) E_{l}(t) .
\end{aligned}
$$

Replacing $t=t_{1}$ and $t+1=t_{2}$ in (93), we have

$$
\begin{aligned}
\int_{t_{1}}^{t_{2}} K(t)\left|u_{l}^{\prime}(t)\right|^{2} & \leq\|K\|_{\infty} \int_{t_{1}}^{t_{2}}\left|u_{l}^{\prime}(t)\right|^{2} d t \\
& \leq \frac{\|K\|_{\infty}}{d_{1}} D_{l}^{2}(t) .
\end{aligned}
$$

Adding (101) and (102), we obtain

$$
\begin{aligned}
\int_{t_{1}}^{t_{2}}\left[K(t)\left|u_{l}^{\prime}(t)\right|^{2}+\left\|u_{l}(t)\right\|^{2}+\left|u_{l}(t)\right|_{L^{\sigma+2}(\Omega)}^{\sigma+2}\right] d t \\
\leq N_{7} \int_{t_{1}}^{t_{2}}\left\|u_{l}(t)\right\|^{2} d t+\left(\frac{\|K\|_{\infty}+N_{5}+N_{6}}{d_{1}}\right) D_{l}^{2}(t) \\
\quad+\frac{2\left(1 / \sqrt{\lambda_{1}}\right)\|K\|_{\infty}}{\sqrt{d_{1}}} E_{l}(t),
\end{aligned}
$$

and this implies

$$
\begin{aligned}
& \int_{t_{1}}^{t_{2}}\left[K(t)\left|u_{l}^{\prime}(t)\right|^{2}+\left(1-N_{7}\right)\left\|u_{l}(t)\right\|^{2}+\left|u_{l}(t)\right|_{L^{\sigma+2}(\Omega)}^{\sigma+2}\right] d t \\
& \quad \leq N_{8} D_{l}^{2}(t)+N_{9} E_{l}(t),
\end{aligned}
$$

where

$$
\begin{aligned}
& N_{8}=\frac{\|K\|_{\infty}+N_{5}+N_{6}}{d_{1}}, \\
& N_{9}=\frac{2\left(1 / \sqrt{\lambda_{1}}\right)\|K\|_{\infty}}{\sqrt{d_{1}}} .
\end{aligned}
$$

The hypothesis (H11) yields $\|b\|_{\infty}<21 \lambda_{1} / 32$ and as $1-N_{7}>0$ then

$$
\begin{aligned}
& \int_{t_{1}}^{t_{2}}\left[\frac{1}{2} K(t)\left|u_{l}^{\prime}(t)\right|^{2}+\frac{1}{2}\left\|u_{l}(t)\right\|^{2}+\frac{1}{\sigma+2}\left|u_{l}(t)\right|_{L^{\sigma+2}(\Omega)}^{\sigma+2}\right] d t \\
& \quad \leq N_{10} D_{l}^{2}(t)+N_{11} E_{l}(t) .
\end{aligned}
$$

Since $1 /(\sigma+2)<1, N_{10}=N_{8} / M$ and $N_{11}=N_{9} / M$, where $M=\min \left\{1,1-N_{7}\right\}>0$.

From (106), we obtain

$$
\int_{t_{1}}^{t_{2}} E_{l}(t) d t \leq N_{10} D_{l}^{2}(t)+N_{11} E_{l}(t) .
$$

Note that by hypothesis (H11), $N_{11}<1 / 4$, thus from (107), we find

$$
\int_{t_{1}}^{t_{2}} E_{l}(t) d t \leq N_{10} D_{l}^{2}(t)+\frac{1}{4} E_{l}(t) .
$$

Again, by the Mean Value Theorem there exists $t^{*} \in\left(t_{1}, t_{2}\right)$, such that

$$
\int_{t_{1}}^{t_{2}} E_{l}(s) d s=E_{l}\left(t^{*}\right)\left(t_{2}-t_{1}\right) \geq \frac{1}{2} E_{l}\left(t^{*}\right) .
$$

Integrating (76) from $t^{*}$ to $t$ and using (77) and (93), it follows that

$$
\begin{aligned}
E_{l}(t) \leq & E_{l}\left(t^{*}\right)+N_{1} \int_{t^{*}}^{t}\left|u_{l}^{\prime}(s)\right|^{2} d s \\
& +N_{2} \int_{t^{*}}^{t}\left|u_{l}^{\prime}(s)\right|_{\Gamma_{1}}^{2} d s \\
\leq & E_{l}\left(t^{*}\right)+\frac{N_{1}}{d_{1}} D_{l}^{2}(t)+\frac{N_{2}}{d_{1}} D_{l}^{2}(t) .
\end{aligned}
$$

Substituting (109) in (110), we get

$$
E_{l}(t) \leq 2 \int_{t_{1}}^{t_{2}} E_{l}(s) d s+\left(\frac{N_{1}}{d_{1}}+\frac{N_{2}}{d_{1}}\right) D_{l}^{2}(t) .
$$

Now, substituting (108) in (111), we obtain

$$
\begin{aligned}
& E_{l}(t) \leq \frac{1}{2} E_{l}(t)+\left(2 N_{10}+\frac{N_{1}}{d_{1}}+\frac{N_{2}}{d_{1}}\right) D_{l}^{2}(t), \\
& E_{l}(t) \leq 2\left(2 N_{10}+\frac{N_{1}}{d_{1}}+\frac{N_{2}}{d_{1}}\right) D_{l}^{2}(t) .
\end{aligned}
$$

As $E_{l}(t)$ is decreasing, the inequality (113) provides that

$$
\sup _{t \leq s \leq t+1} E_{l}(s) \leq N_{12}\left[E_{l}(t)-E_{l}(t+1)\right],
$$

where

$$
N_{12}=2\left(2 N_{10}+\frac{N_{1}}{d_{1}}+\frac{N_{2}}{d_{1}}\right)>0 .
$$

Thus, it follows using (114) and from Nakao's Lemma, (see [23]), that

$$
E_{l}(t) \leq c e^{-w t}, \quad t \geq 1 .
$$

Taking the $\lim \inf$ as $l \rightarrow \infty$ in (116), we obtain

$$
E(t) \leq c e^{-w t}, \quad \forall t \geq 1,
$$

where $c$ is positive constant.

\section{Conflict of Interests}

The authors report that there is no conflict of interests in the publication of this paper. 


\section{References}

[1] V. Komornik and E. Zuazua, "A direct method for the boundary stabilization of the wave equation," Journal de Mathématiques Pures et Appliquées, vol. 69, no. 1, pp. 33-54, 1990.

[2] M. Milla Miranda and L. A. Medeiros, "On a boundary value problem for wave equations: existence uniqueness-asymptotic behavior," Revista de Matemáticas Aplicadas, vol. 17, no. 2, pp. 47-73, 1996.

[3] F. D. Araruna and A. B. Maciel, "Existence and boundary stabilization of the semilinear wave equation," Nonlinear Analysis: Theory, Methods \& Applications, vol. 67, no. 4, pp. 1288-1305, 2007.

[4] M. M. Cavalcanti, V. N. Domingos Cavalcanti, J. A. Soriano, and L. A. Medeiros, "On the existence and the uniform decay of a hyperbolic equation with non-linear boundary conditions," Southeast Asian Bulletin of Mathematics, vol. 24, no. 2, pp. 183$199,2000$.

[5] M. M. Cavalcanti, V. N. Domingos Cavalcanti, J. S. Prates Filho, and J. A. Soriano, "Existence and uniform decay of solutions of a degenerate equation with nonlinear boundary damping and boundary memory source term," Nonlinear Analysis, vol. 38, no. 3, pp. 281-294, 1999.

[6] M. M. Cavalcanti, V. N. Domingos Cavalcanti, and P. Martinez, "Existence and decay rate estimates for the wave equation with nonlinear boundary damping and source term," Journal of Differential Equations, vol. 203, no. 1, pp. 119-158, 2004.

[7] I. Lasiecka and D. Tataru, "Uniform boundary stabilization of semilinear wave equations with nonlinear boundary damping," Differential and Integral Equations, vol. 6, no. 3, pp. 507-533, 1993.

[8] M. Milla Miranda and L. P. San Gil Jutuca, "Existence and boundary stabilization of solutions for the Kirchhoff equation," Communications in Partial Differential Equations, vol. 24, no. 910, pp. 1759-1800, 1999.

[9] A. T. Lourêdo and M. M. Miranda, "Local solutions for a coupled system of Kirchhoff type," Nonlinear Analysis: Theory, Methods \& Applications, vol. 74, no. 18, pp. 7094-7110, 2011.

[10] A. T. Lourêdo and M. M. Miranda, "Nonlinear boundary dissipation for a coupled system of Klein-Gordon equations," Electronic Journal of Differential Equations, vol. 120, pp. 1-19, 2010.

[11] A. T. Lourêdo, M. A. Ferreira de Araújo, and M. M. Miranda, "On a nonlinear wave equation with boundary damping," Mathematical Methods in the Applied Sciences, vol. 37, no. 9, pp. 1278-1302, 2014.

[12] J. Y. Park and J. R. Kang, "Existence, uniqueness and uniform decay for the non-linear degenerate equation with memory condition at the boundary," Applied Mathematics and Computation, vol. 202, no. 2, pp. 481-488, 2008.

[13] M. de Lima Santos and F. Junior, "A boundary condition with memory for Kirchhoff plates equations," Applied Mathematics and Computation, vol. 148, no. 2, pp. 475-496, 2004.

[14] D. C. Pereira, "Existence, uniqueness and asymptotic behavior for solutions of the nonlinear beam equation," Nonlinear Analysis: Theory, Methods \& Applications, vol. 14, no. 8, pp. 613-623, 1990.

[15] J.-L. Lions, Equations aux Dérivées Partielles-Interpolation, Volume I, Oeuvres choisies de Jacques-Louis Lions, SMAI, EDP Sciences, Paris, France, 2003.
[16] W. A. Strauss, "On weak solutions of semilinear hyperbolic equations," Anais da Academia Brasileira de Ciências, vol. 42, pp. 645-651, 1970.

[17] H. Brezis and T. Cazenave, Nonlinear Evolution Equations, Universidade Federal do Rio de Janeiro, Rio de Janeiro, Brazil, 1994.

[18] M. Marcus and V. J. Mizel, "Every superposition operator mapping one Sobolev space into another is continuous," Journal of Functional Analysis, vol. 33, no. 2, pp. 217-229, 1979.

[19] J. L. Lions, Quelques Méthodes de Résolution des Problemes aux Limites Non Linéaires, Dunod/Gauthier-Villars, Paris, France, 1969.

[20] J. Simon, "Compact sets in the space $L^{p}(0, T ; B)$," Annali di Matematica Pura ed Applicata Série IV, vol. 146, pp. 65-96, 1987.

[21] L. A. Medeiros and M. AMilla Miranda, Espaços de Sobolev (Iniciação aos Problemas Eliticos Não Homogêneos), Editora IMUFRJ, 5th edition, 2006.

[22] J. L. Lions and E. Magenes, Problèmes aux Limites Non Homogènes et Applications, vol. 1, Dunod, Paris, France, 1968.

[23] M. Nakao, "Decay of solutions of the wave equation with a local nonlinear dissipation," Mathematische Annalen, vol. 305, no. 3, pp. 403-417, 1996. 


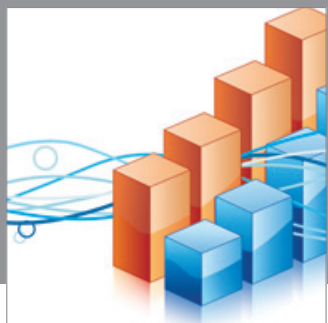

Advances in

Operations Research

mansans

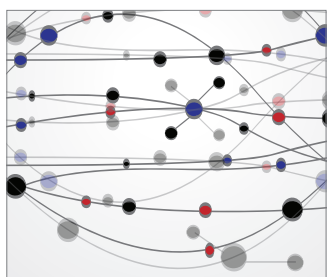

The Scientific World Journal
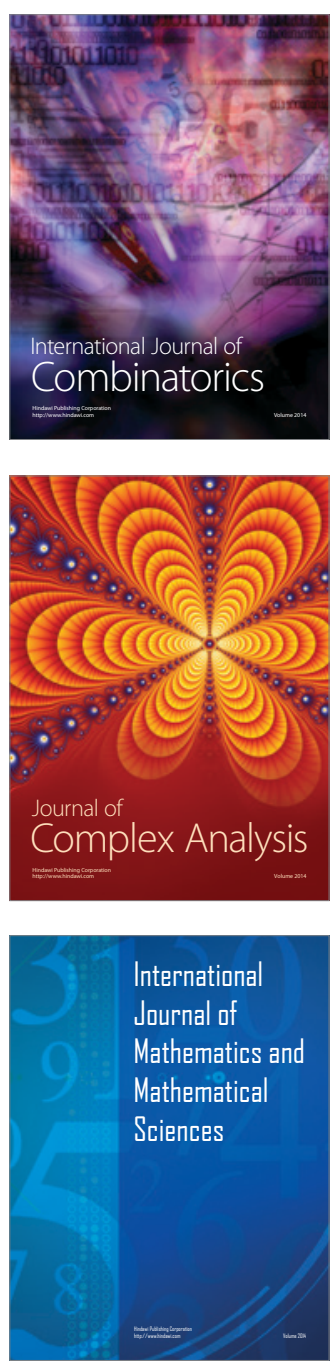
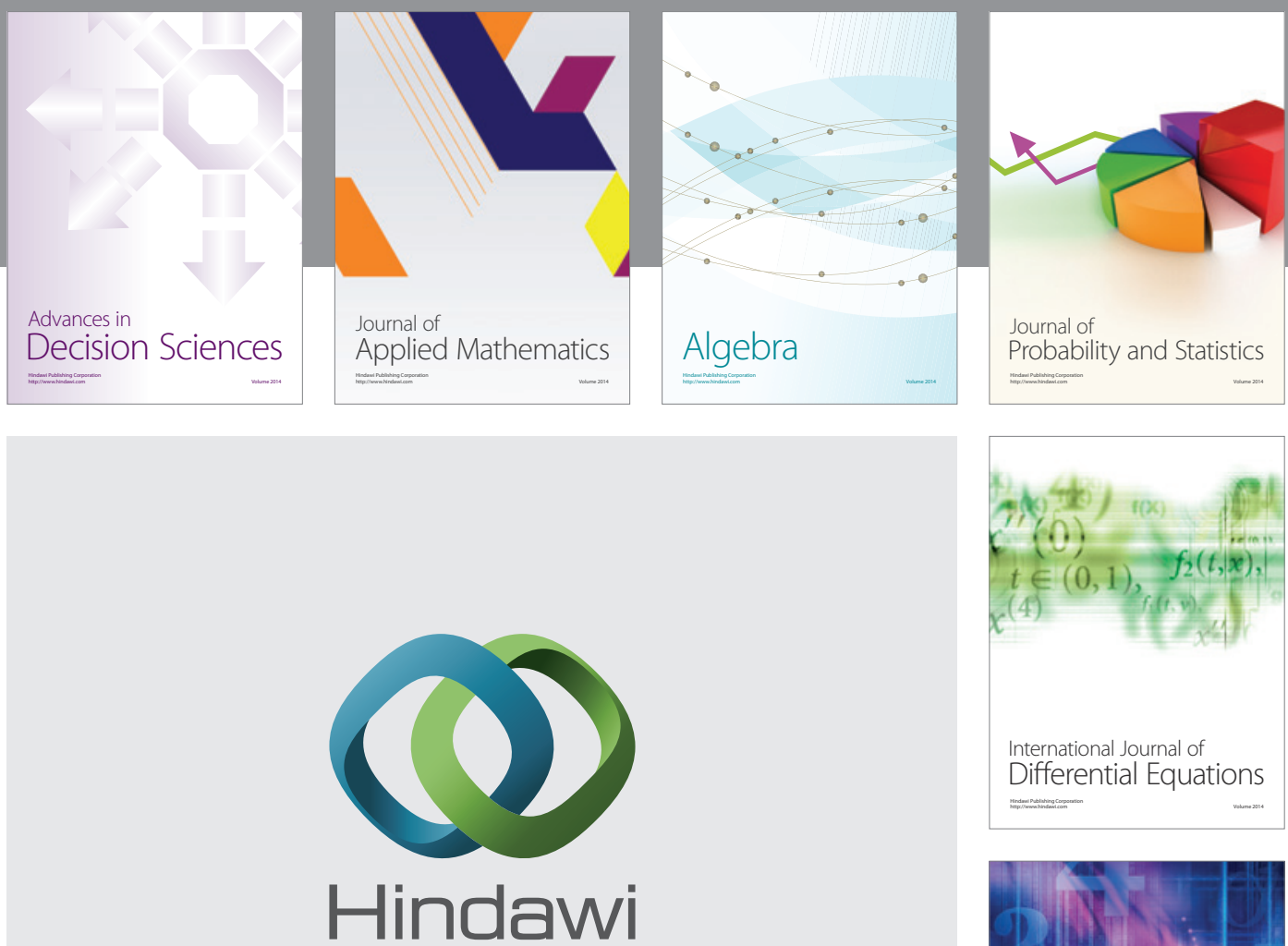

Submit your manuscripts at http://www.hindawi.com
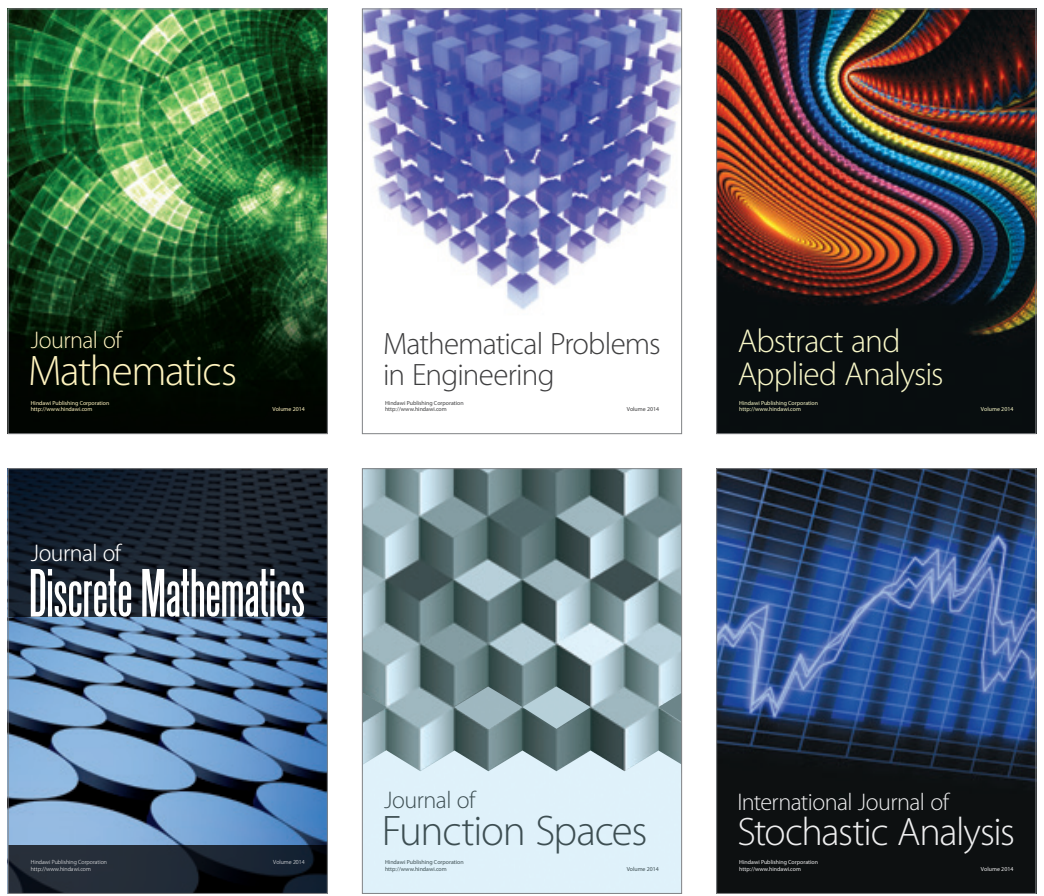

Journal of

Function Spaces

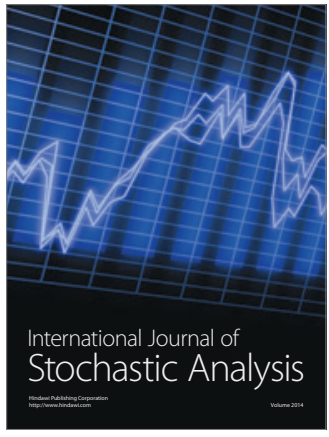

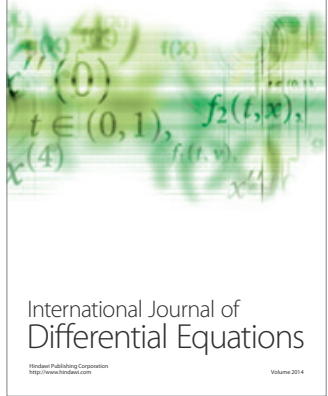
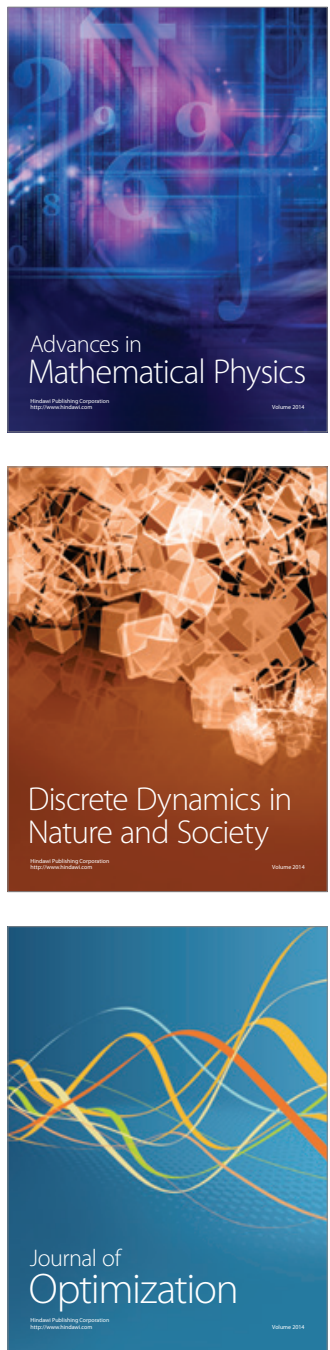